ARTICLE

https://doi.org/10.1038/s41467-019-13157-7

\title{
Renewed proliferation in adult mouse cochlea and regeneration of hair cells
}

\author{
Yilai Shu',2,3,4,10, Wenyan Li1,2,3,4,10, Mingqian Huang ${ }^{1,2,10}$, Yi-Zhou Quan 1,2,10, Deborah Scheffer ${ }^{1,2,5}$, \\ Chunjie Tian ${ }^{1,2}$, Yong Tao ${ }^{1,2}$, Xuezhong Liu ${ }^{6}$, Konrad Hochedlinger (1) 7,8,9, Artur A. Indzhykulian ${ }^{1,2}$, \\ Zhengmin Wang ${ }^{3,4}$, Huawei $\mathrm{Li}^{3,4} \&$ Zheng-Yi Chen (i) ${ }^{1,2 \star}$
}

The adult mammalian inner ear lacks the capacity to divide or regenerate. Damage to inner ear generally leads to permanent hearing loss in humans. Here, we present that reprogramming of the adult inner ear induces renewed proliferation and regeneration of inner ear cell types. Co-activation of cell cycle activator Myc and inner ear progenitor gene Notch1 induces robust proliferation of diverse adult cochlear sensory epithelial cell types. Transient MYC and NOTCH activities enable adult supporting cells to respond to transcription factor Atoh1 and efficiently transdifferentiate into hair cell-like cells. Furthermore, we uncover that mTOR pathway participates in MYC/NOTCH-mediated proliferation and regeneration. These regenerated hair cell-like cells take up the styryl dye FM1-43 and are likely to form connections with adult spiral ganglion neurons, supporting that Myc and Notch1 co-activation is sufficient to reprogram fully mature supporting cells to proliferate and regenerate hair celllike cells in adult mammalian auditory organs.

\footnotetext{
${ }^{1}$ Department of Otolaryngology-Head and Neck Surgery, Graduate Program in Speech and Hearing Bioscience and Techology and Program in Neuroscience, Harvard Medical School, Boston, MA 02115, USA. ²Eaton-Peabody Laboratory, Massachusetts Eye and Ear Infirmary, 243 Charles St., Boston, MA 02114, USA. ${ }^{3}$ ENT Institute and Otorhinolaryngology Department of the Affiliated Eye and ENT Hospital, State Key Laboratory of Medical Neurobiology, Institutes of Biomedcial Sciences, Fudan University, 200031 Shanghai, China. ${ }^{4}$ NHC Key Laboratory of Hearing Medicine, Fudan University, Shanghai 200031, China.

${ }^{5}$ Department of Neurobiology and Howard Hughes Medical Institute, Harvard Medical School, Boston, MA 02115, USA. ${ }^{6}$ Department of Otolaryngology, University of Miami School of Medicine, Miami, FL 33136, USA. ${ }^{7}$ Department of Molecular Biology, Cancer Center and Center for Regenerative Medicine, Massachusetts General Hospital, Boston, MA 02114, USA. ${ }^{8}$ Department of Stem Cell and Regenerative Biology and Harvard Stem Cell Institute, Cambridge, MA 02138, USA. ${ }^{9}$ Howard Hughes Medical Institute, Chevy Chase, MD 20815, USA. ${ }^{10}$ These authors contributed equally: Yilai Shu, Wenyan Li, Mingqian Huang, Yi-Zhou Quan *email: Zheng-Yi_Chen@meei.harvard.edu
} 
O ne of the most common human sensory disorders, hearing loss affects approximately one in 500 newborns and more than half of the population over 70 years of age $^{1,2}$. Despite its prevalence, there remains no available pharmacological therapies to treat hearing loss. Loss of hair cells (HCs), the inner ear sensory cells that detect sound and sense balance, is a major cause of hearing loss and vestibular dysfunction in humans. In lower vertebrates such as birds, fish, and amphibians, HC loss triggers supporting cells (SCs) to re-enter the cell cycle ${ }^{1-4}$. Proliferating SCs then transdifferentiate into new $\mathrm{HCs}$, resulting in the recovery of hearing and vestibular functions ${ }^{5}$. In contrast, the mature mammalian cochlea completely lacks the capacity to spontaneously proliferate or regenerate HCs, and has very limited regeneration potential in the vestibular system $^{6,7}$.

To recover from deafness due to HC loss, reprogramming of adult SCs is likely necessary for renewed proliferation and transdifferentiation of SCs to HCs (SC-to-HC). In lower vertebrates, these two events occur spontaneously ${ }^{1,2,8}$, however, the underlying mechanisms remain largely unknown. In culture, neonatal mouse inner ear SCs retain the capacity to proliferate and transdifferentiate into $\mathrm{HCs}^{9}$, yet, this capacity is lost in the adult inner ear. As SCs serve essential roles in hearing and balance and deficits in SCs can result in deafness ${ }^{10}$, an ideal approach to regenerate $\mathrm{HCs}$ by SC transdifferentiation would require replacement of lost SCs. Thus, renewed proliferation is likely best suited to achieve a balance between $\mathrm{HC}$ regeneration and maintenance of the SC population.

Multiple cell cycle genes, including $R b 1, C d k n 1 b$ (p27Kip1), Cdkn2d (p19Ink4d), and Cdkn1a (p21Cip1) ${ }^{11-16}$, have been studied in induction of proliferation in the mammalian inner ear, however, none were sufficient in inducing proliferation in the adult cochlea. In the young mammalian inner ear, SC-to-HC transdifferentiation can be induced by overexpression of $\mathrm{HC}$ fatedetermining transcription factor, Atoh $1^{17}$. An early study provided evidence that Atoh1 overexpression had limited but similar effects in the adult mammalian cochlea, however, subsequent studies failed to reproduce the essential findings ${ }^{18-22}$. It is therefore suggested that, in the adult inner ear, overexpression of Atoh1 in SCs alone is inefficient in promoting HC regeneration. To recapture the capacity to respond to $\mathrm{HC}$ induction signals, it is likely that mature SCs need to first regain the properties of their younger biological selves.

To identify potential reprogramming factors in the adult mammalian inner ear, we began by studying chick and zebrafish $\mathrm{HC}$ regeneration models and uncovered that reactivation of $M y c$ is a major event that leads to cell cycle re-entry ${ }^{23}$, suggesting that a similar mechanism could induce proliferation in the mammalian inner ear. Additional studies have shown that overexpression of Notch1, a receptor important in mammalian inner ear early development and patterning, is sufficient to induce formation of the prosensory domain of the developing mouse otocyst; ${ }^{24,25}$ indicating the prominent role of Notch 1 in conferring prosensory domain properties. We hypothesize that the combined action of MYC and NOTCH1 may be sufficient to reprogram adult mouse inner ear cells for cell cycle re-entry and the reprogrammed SCs may regain the properties enabling them to transdifferentiate into $\mathrm{HCs}$ in the presence of induction signals.

In this study, by adenovirus-mediated delivery and inducible transgenic mouse models, we demonstrate the proliferation of both HCs and SCs by combined Notch1 and Myc activation in in vitro and in vivo inner ear adult mouse models. These proliferating mature SCs and HCs maintain their respective identities. Moreover, when presented with HC induction signals, reprogrammed adult SCs transdifferentiate into HC-like cells both in vitro and in vivo. We identify the mTOR pathway as downstream of $M y c /$ Notch1 activation and therefore a required player in proliferation and SC-to-HC transdifferentiation in the adult cochlea. Finally, our data suggest that regenerated HC-like cells likely possess functional transduction channels and are able to form connections with adult auditory neurons.

\section{Results}

$M y c /$ Notch1 co-activation induces division in adult inner ear. In lower vertebrates, SC proliferation and transdifferentiation are major mechanisms involved in $\mathrm{HC}$ regeneration ${ }^{8}$. In zebrafish model after HC damage, reactivation of $c-m y c(m y c)$ is necessary for renewed SC proliferation ${ }^{23}$. Further Notch is activated during zebrafish $\mathrm{HC}$ regeneration ${ }^{26}$. To evaluate the direct role of $M y c$ in renewed proliferation in the mouse inner ear, we used the cochleostomy technique to inject adenovirus carrying human MYC (ad- $M y c)^{27}$ into the mouse cochlear scala media, and studied proliferation via BrdU incorporation. BrdU was detected in postnatal day 7 (P7) cochlear SCs $\left(\mathrm{SOX}^{+}\right)$(Supplementary Fig. 1a-d), but not in the adult mouse cochlea (Supplementary Fig. 1f). The cochleostomy procedure in adults damages a majority of the outer hair cells (OHCs) around the injection site, consistent with our previous study ${ }^{28}$. To study the independent effect of Notch1 activation, we injected an adenovirus carrying Cre recombinase gene (ad-Cre) into the cochlea of adult RosaNICD transgenic mice ${ }^{29}$ which carry a floxed stop codon that blocks expression of the Notch1 intracellular domain (NICD). NICD activation alone did not induce proliferation (Supplementary Fig. 1g).

We hypothesized that reprogramming by combined action of inner ear progenitor genes and cell cycle activators is necessary to induce proliferation in adult cochlea. We determined the combined effect of $M y c$ and Notch1 co-activation by injecting a mixture of ad-Myc/ad-Cre virus into fully mature (6 weeks) RosaNICD cochlea, followed by BrdU intraperitoneal (i.p.) injection in vivo (Fig. 1a). Checking at two different time points, four and 35 days after injection, we found proliferating inner hair cells (IHCs) $\left(\mathrm{MYO}^{+} \mathrm{A}^{+} / \mathrm{BrdU}^{+}\right)$and $\mathrm{SCs}\left(\mathrm{SOX} 2^{+} / \mathrm{BrdU}^{+}\right)$at the injection site in the injected cochlea (Fig. $1 b-i$ and $n-o$ ). In comparison, no proliferating cells were found in the ad-V5injected control adult Rosa-NICD cochlea (Fig. 1j-o; Supplementary Fig. 1j) or in the uninjected cochlea (Supplementary Fig. 1h).

Regeneration studies in the adult mammalian inner ear are particularly challenging due to the lack of an efficient in vitro system that allows such studies. To overcome this limitation and effectively study reprogramming and proliferation in adult cochleae, we established a system of adult whole cochlea explant culture (Supplementary Fig. 2). This was achieved by culturing adult cochlea encased in bone with the opening in the apex and the base (see Methods for details). In this model, while all OHCs and a majority of IHCs die rapidly in culture, a majority of SCs $\left(\mathrm{SOX}^{+}\right)$survive for over two weeks while maintaining an intact overall cochlear structure (Supplementary Fig. 2).

Cell division is marked by a series of distinct cellular events. To study cell cycle, we cultured adult Rosa-NICD cochleae and infected them with ad-Myc/ad-Cre (Fig. 2a). We identified SCs in mitosis by mitotic figures and MKI67 labeling (Fig. 2b-d, h). Dividing SCs were labeled with mitosis (phosphorylated histone H3, pH3) (Fig. 2e, i) and cytokinesis (Aurora B, AURKB; a-tubulin, TUBA4A) (Fig. 2f, g) markers. We quantified the number of SCs $\left(\mathrm{SOX}_{2}{ }^{+}\right)$seven days after ad-Myc/ad-Cre infection in Rosa-NICD cochleae in the mid-apical region, which included outer pillar cells, Deiters' cells, Hensen cells and Claudius' cells (Fig. 1a), and found a significant increase in the 
a

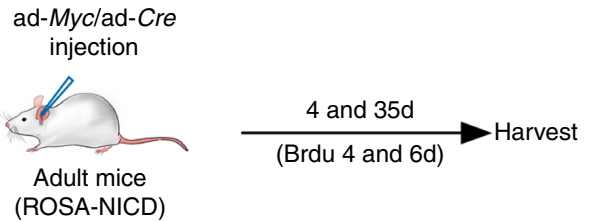

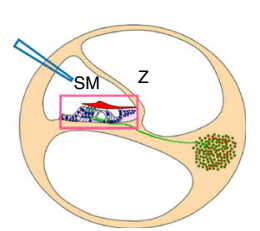

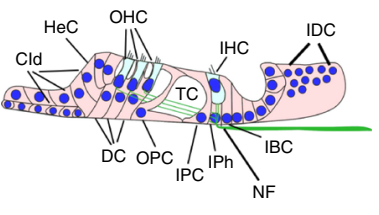

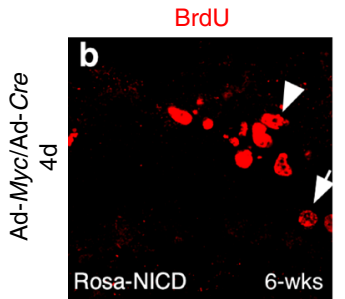
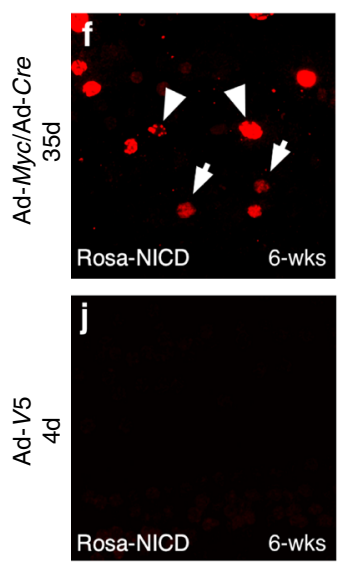

MYO7A
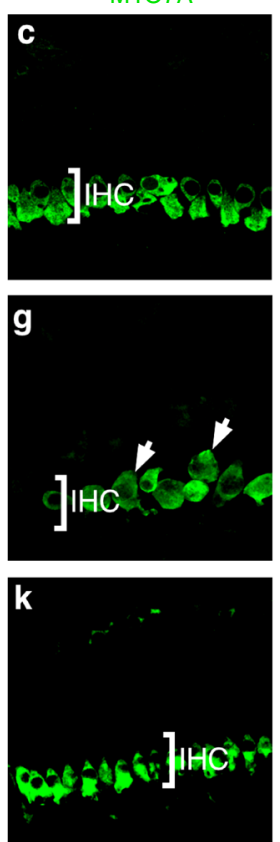

SOX2
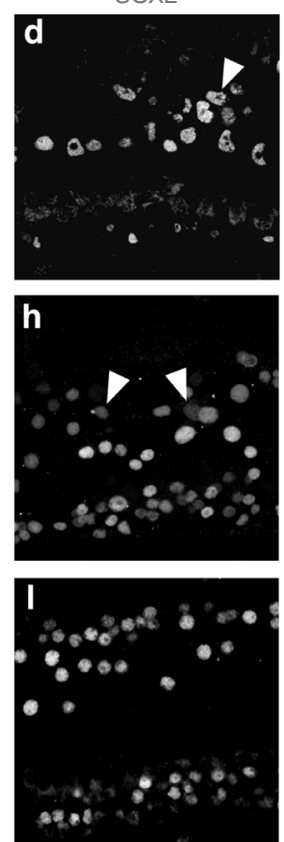

Merge/DAPI
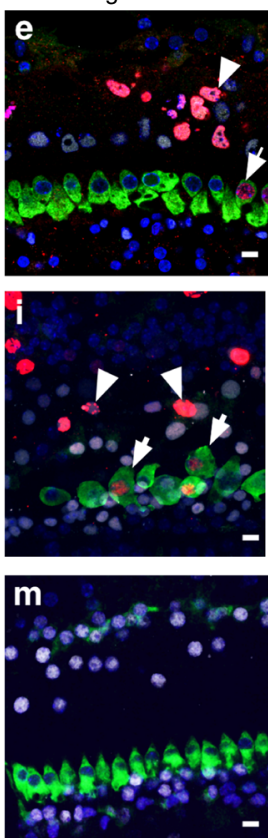

n

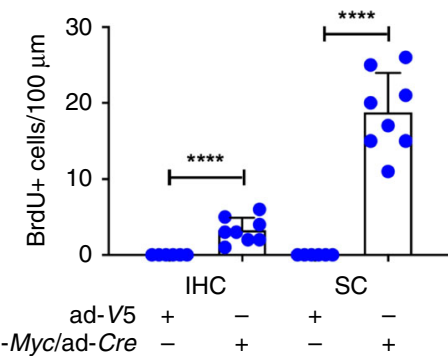

o

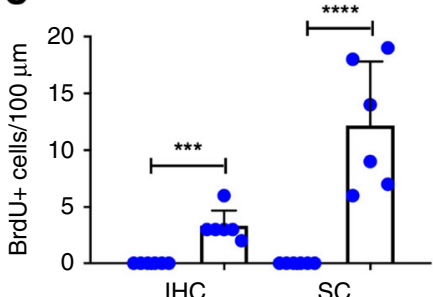

ad- $V 5$ ad-Myclad-Cre

Fig. $1 \mathrm{Myc}$ and NICD co-activation induces proliferation in adult mouse cochlea in vivo. a A diagram illustrating the procedure of ad-Myc/ad-Cre injection in adult Rosa-NICD cochlea (left). A diagram depicts injection into the scala media (SM) of adult cochlea by cochleostomy (middle). Enlarged inset of a cross section shows cochlear structure and cell subtypes (right). Cld: Claudius' cells; HeC: Hensen cells; OHC: outer hair cells; IHC: inner hair cells; IDC: interdental cells; DC; Deiters' cells; OPC: outer pillar cells; TC: tunnel of Corti; IPC: inner pillar cells; IPh: inner phalangeal cells; NF: neuro fibers of auditory neurons; and IBC: inner cells. b-i Four (b-e) and 35 (f-i) days after injecting ad-Myc/ad-Cre mixture to the adult (6-week-old) Rosa-NICD cochleae in vivo, proliferating IHCs (MYO7A+/BrdU + , arrows) and SCs (SOX2+/BrdU + , arrowheads) were detected. $\mathbf{j}-\mathbf{m}$ Four days after control ad-V5 in vivo injection into the adult Rosa-NICD cochleae, no proliferating cell was detected. $\mathbf{n}, \mathbf{o}$ Quantification and comparison of number and percentage of BrdU+ IHCs and SCs in adult Rosa-NICD cochleae infected with ad-Myc/ad-Cre in vivo for four days (n) or 35 days (o) in the mid-base turn, compared to the ad-V5-injected adult Rosa-NICD cochleae. ${ }^{\star \star \star} p<0.001,{ }^{\star \star \star \star} p<0.0001$, two-tailed unpaired Student's $t$-test. Error bar, mean \pm s.d.; $n=8$ for ad-Myc/ad-Cre groups, $n=6$ for control groups in (n); $n=6$ for each group in (0). $n$ is the number of biologically independent samples. Scale bars: $10 \mu \mathrm{m}$. Source data are provided as a Source Data file.

total number of $\mathrm{BrdU}^{+}$cells and SCs in ad-Myc/ad-Cre-infected cochleae compared to the ad-V5-infected controls (Fig. 2j, k).

To characterize IHC proliferation in response to $M y c / N I C D$ co-activation, we injected ad-Myc/ad-Cre into adult Rosa-NICD mouse cochlea in vivo via cochleostomy. Some IHCs were induced to divide $\left(\mathrm{BrdU}^{+}\right.$doublets) with mitotic figures (labeled by MKI67 and pH3) (Fig. 2l-n, q-s). Thirty-five days after injection, stereocilia in proliferating IHCs were still present (Supplementary Fig. 1i, arrow). Injected adult cochleae showed dividing IHCs with enlarged cell bodies (Fig. 20, p), consistent with $M y c$ overexpression shown to increase cell $\operatorname{size}^{30}$. We counted the number of IHCs and found ad-Myc/ad-Cre-injected adult ROSA-NICD cochlea had more IHCs compared to the adV5-injected control cochleae at the injection site (Fig. 2t).

To determine whether proliferation is the direct result of coactivation of $M y c$ and NICD, we infected cultured adult wild type cochlea explants with a mixture of ad- $M y c$ and adenovirus carrying a V5-epitope-tagged NICD (ad-NICD-V5) ${ }^{31}$ (Supplementary Fig. 3a). We found that a majority of $\mathrm{BrdU}^{+}$cells were double positive for MYC and NICD (V5) $(89 \pm 5 \%, n=4$ from biologically independent samples; mean \pm s.e.m.), with the remaining being $\mathrm{MYC}^{+}$only $(5.6 \pm 3.1 \%)$ or $\mathrm{NICD}^{+}$only $(3.1 \pm 0.8 \%)$, or negative for both $(2.1 \pm 1.4 \%)$ (Supplementary Fig. 3b). Semi-quantitative RT-PCR showed an up-regulation of Notch1/NICD and Notch1 target Hes5 after ad-Myc/ad-Cre infection of the cultured adult Rosa-NICD cochlea (Supplementary Fig. 3d). Together, this provides sufficient evidence that Myc/Notch1 co-activation induces adult cochlear SCs and IHCs to re-enter and complete the cell cycle, resulting in increased SC and IHC numbers.

To study the origin of proliferating cells in adult cochlea, we performed lineage tracing using inducible Sox2-promoter-driven Cre mice (Sox2-CreER) ${ }^{32}$ crossed with tdTomato (tdT) reporter mice. In resulting Sox2-CreER/tdT $/ \mathrm{f} / \mathrm{f}$ mice, exposure to tamoxifen $(\mathrm{Tm})$ induces tdT expression permanently and specifically in $\mathrm{SCs}^{33}$. To better understand the origin of proliferating HCs, we injected cochleae of adult Sox2-CreER/tdT $\mathrm{T}^{\mathrm{f} / \mathrm{f}}$ mice with either ad-V5 or an ad-Myc/ad-NICD mixture after administering the Tm by i.p. injection (Fig. 3a). In the control ad-V5-injected Sox2-CreER/tdT $T^{\mathrm{t} / \mathrm{f}}$ cochleae, SCs $\left(\mathrm{SOX2}^{+}\right)$and IHCs $\left(\mathrm{MYO}^{+} \mathrm{A}^{+}\right)$were $\mathrm{tdT}^{+}$and $\mathrm{tdT}^{-}$, respectively (Fig. 3b). In the ad-Myc/ad-NICD-injected cochleae, dividing HCs were detected and all were found to be $\operatorname{tdT}^{-}$(Fig. 3c), demonstrating that they were derived from existing IHCs. To study the origin of proliferating SCs, we infected cultured adult Sox $2-C r e E R / t^{2} T^{\mathrm{f} / \mathrm{f}}$ cochlea in vitro with ad-Myc/ad-NICD after 

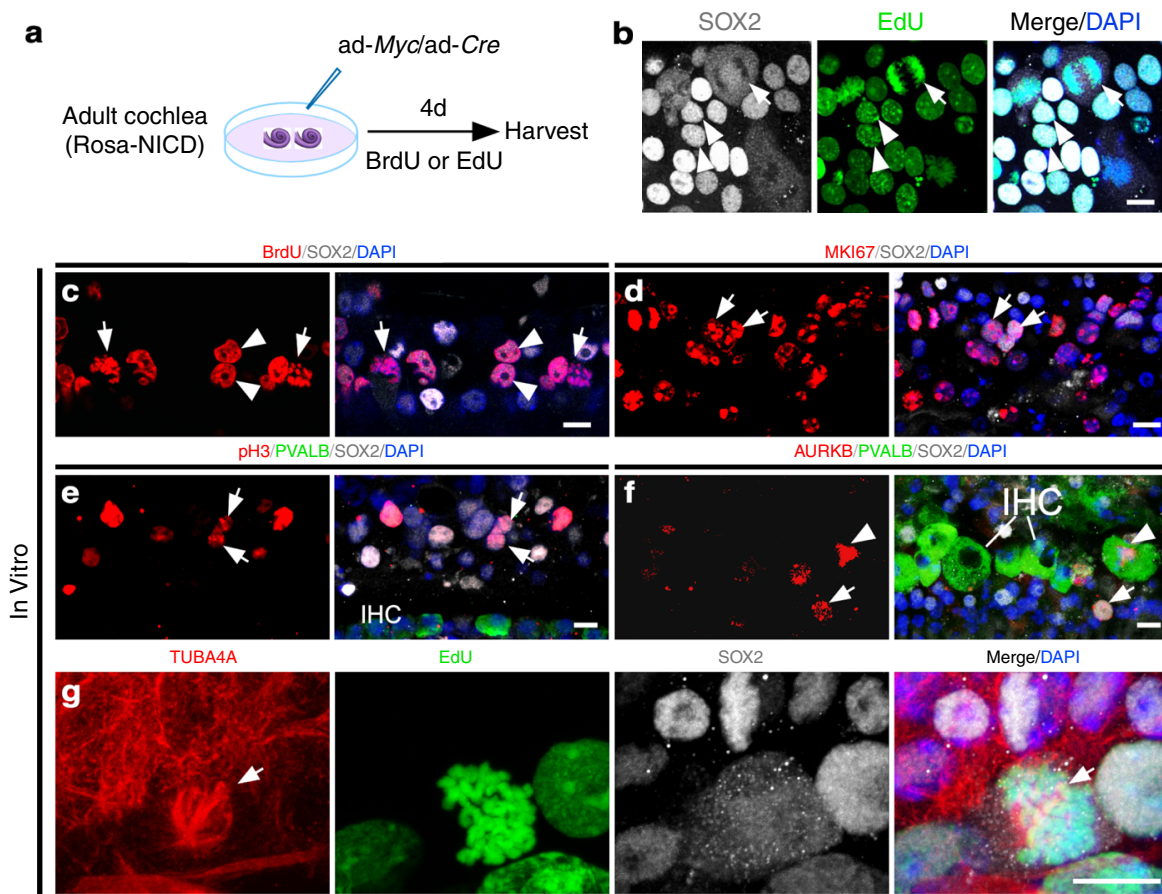

BrdU/SOX2/DAPI
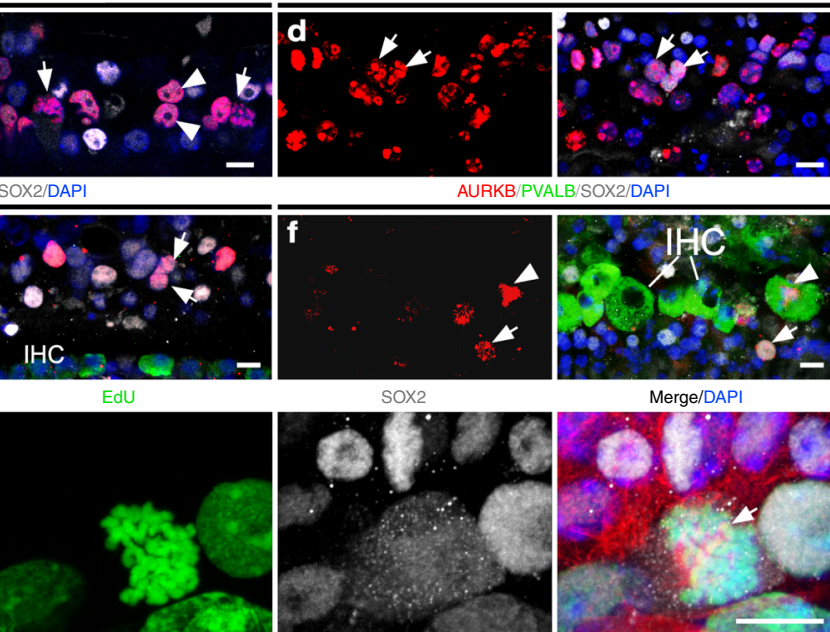

Merge/DAPI
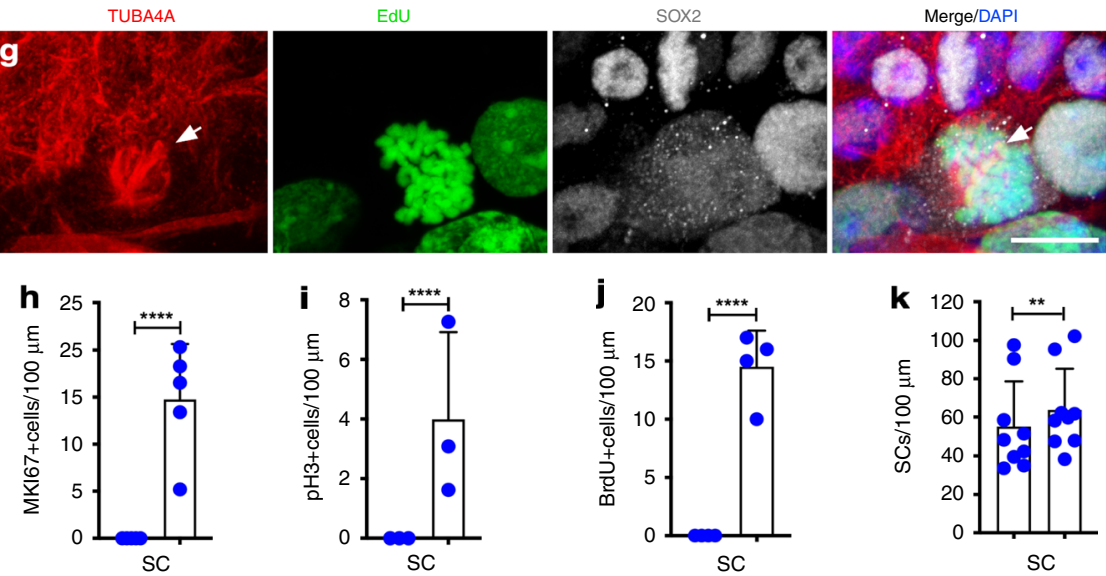

ad- $V 5$ ad-Myc/ad-Cre

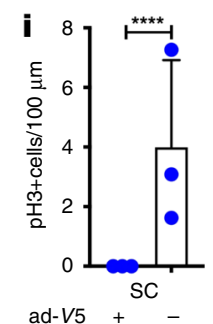

ad- $V 5+-$
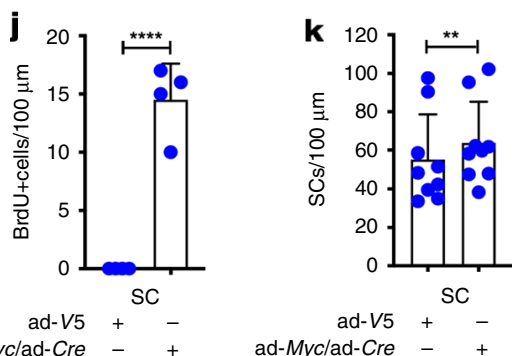

ad-Myc/ad-Cre -

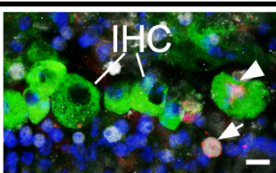

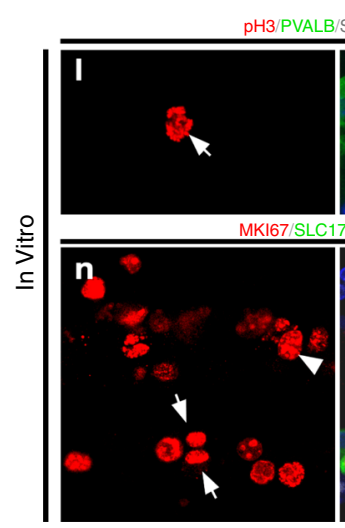

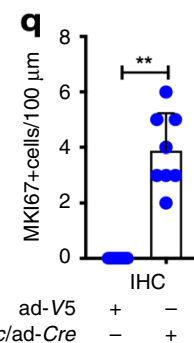
(n) 
Fig. 2 Characterization of proliferation in adult cochlea. a A diagram illustrating the experimental procedure. b-f Four days post ad-Myc/ad-Cre infection of the Rosa-NICD adult (6-week-old) cochlear explant in vitro, SC mitotic figures (b, c, arrows), dividing SC nuclear doublet (b, $\mathbf{c}$, arrowheads), MKI67 ${ }^{+}\left(\mathrm{Ki67} 7^{+}\right) \mathrm{SCs}$ (d, arrows) were seen. $\mathrm{pH}^{+} \mathrm{SCs}(\mathbf{e}$, arrows), AURBK $+\mathrm{SCs}(\mathbf{f}$, arrow) and AURBK+ IHCs (f, arrowhead) were detected. $\mathbf{g}$ Alpha-tubulin labeled dividing SCs $\left(\mathrm{TUBA}_{4} \mathrm{~A}^{+} / \mathrm{SOX}^{+} / \mathrm{EdU}^{+}\right.$) in vitro, with the arrow showing TUBA4A ${ }^{+}$spindle microtubules. h-k Quantification showed an increase in the SCs labeled with MKI67 (h), pH3 (i) and BrdU (j), as well as the total number of SCs (k) in ad-Myc/ad-Cre-infected compared to control ad-V5-infected Rosa-NICD cochleae. ${ }^{\star \star} p<0.01,{ }^{\star \star \star \star} p<0.0001$, two-tailed unpaired $(\mathbf{h}-\mathbf{j})$ and paired (k) Student's t-tests. Error bar, mean \pm s.d.; $\mathbf{h}: n=5 ; \mathbf{i}: n=4 ; \mathbf{j}: n=3 ; \mathbf{k}: n=9$. I-n Four $(\mathbf{I})$ and $\operatorname{six}(\mathbf{m}, \mathbf{n})$ days after ad-Myc/ad-Cre injection into adult Rosa-NICD mouse cochlea in vivo, a pH3 + IHC (I, arrow; arrowhead points to hair bundles), a dividing $\mathrm{IHC}(\mathbf{m})$ with the arrow pointing to the cleavage site between two daughter cells, and MKI67 ${ }^{+} \mathrm{IHCs}\left(\mathbf{n}, \mathrm{SLC17A8} 8^{+} / \mathrm{MKI}^{+} 7^{+}\right.$, arrows point to a pair of IHC doublets) and SCs (n, SOX2+/MKI67+, arrowhead) were detected. o, p Compared to a control ad-V5-injected Rosa-NICD adult cochlea without proliferation (o), the ad-Myc/ad-Cre injected Rosa-NICD cochlea showed IHC proliferation with enlarged cell bodies (p, arrows show EdU + IHC with enlarged bodies), 12 days after injection. $\mathbf{q}, \mathbf{r}$ Quantification showed increase in MKI67 ${ }^{+} \mathrm{IHCs}(\mathbf{q})$ and pH3 ${ }^{+} \mathrm{IHCs}(\mathbf{r})$ in adult Rosa-NICD cochleae injected with ad-Myc/ad-Cre or ad-V5 for 4 days, respectively. ${ }^{\star \star} p<0.01$, two-tailed unpaired Student's $t$-test. Error bar, mean \pm s.d.; $n=8$ for each group in ( $\left.\mathbf{q}\right)$; $n=6$ for each group in ( $\mathbf{r}$ ). $\mathbf{s ,} \mathbf{t}$ Quantification showed increase in $\mathrm{BrdU}+\mathrm{IHCs}(\mathbf{s})$ and the total number of IHCs (t) in adult Rosa-NICD cochleae injected with ad-Myc/ad-Cre and ad-V5 in vivo at six days. ${ }^{\star \star \star \star} p<0.0001$, two-tailed unpaired Student's $t$-test. Error bar, mean \pm s.d.; $n=8$ for each group in (s, $\left.\mathbf{t}\right) . n$ is the number of biologically independent samples. Scale bars: $10 \mu \mathrm{m}$. Source data are provided as a Source Data file.

To assess possible cell death after viral infection, we performed cleaved-caspase-3 (CASP3) labeling using cultured adult RosaNICD cochleae infected by ad-Myc/ad-Cre, as infection rate was more uniform in culture. Compared to ad-GFP-infected control cochleae, ad-Myc/ad-Cre-infected cochleae showed significantly more $\mathrm{CASP}^{+}{ }^{+}$cells in the mid-apex in the sensory epithelial region (Supplementary Fig. 5a-f), supporting that MYC/NICD activation induces apoptosis. While cell death is consistent with high and sustained MYC activity due to adenovirus-mediated overexpression, our subsequent experiment showed that under transient $M y c /$ Notch activation, proliferating cells can survive.

Production of HC-like cells by transient $M y c / N I C D$ activation. In contrast to lower vertebrates, in which HC loss results in SCs dividing and spontaneously transdifferentiating into $\mathrm{HCs}^{1-4}$, after $M y c / N I C D$ induced SC proliferation in vivo, we did not detect new HCs. There were no new HCs in the OHC region where the surgical procedure by cochleostomy killed all $\mathrm{OHCs}^{28}$ (Fig. 1b-i) or SC-derived HCs in the lineage-tracing study (Fig. 3), indicating the lack of spontaneous transdifferentiation from SCs to HCs upon cell cycle re-entry. Despite the evidence that overexpression of Atoh1 induces cochlear SCs to transdifferentiate into HCs in neonatal and embryonic mice ${ }^{17,34}$, such transdifferentiation by Atoh1 overexpression is extremely inefficient at the adult stage ${ }^{21}$. To determine if Atoh1 promotes dividing adult SCs to transdifferentiate to HCs, we performed a triple infection with mixed ad-Myc/ad-Cre/adAtoh 1 in the cultured adult Rosa-NICD cochlea and analyzed HC regeneration. To our surprise, no new HCs were generated in cultured cochlea after triple infection (Supplementary Fig. 6).

The lack of SC-to-HC transdifferentiation, either spontaneously or in response to Atoh1 overexpression, could be due to sustained $M y c / N I C D$ co-activation that promotes proliferation but prevents transdifferentiation ${ }^{35}$. To promote SC-to-HC transdifferentiation, it may be necessary to transiently activate $M y c / N I C D$ to reprogram SCs and then follow with HC induction signals.

To activate $M y c / N o t c h 1$ transiently, we created a doxycycline (Dox)-inducible transgenic mouse line by crossing Rosa26promoter-driven reverse tetracycline-controlled transactivator (rtTA) transgenic mice with tetracycline-response-elementcontrolled $M y c$ and NICD mice (tet- $M y c$ and tet-NICD), creating an rtTA/tet-Myc/tet-NICD inducible model. Using explant culture, we showed that $M y c$ and NICD expression are tightly and reversibly controlled by the exposure and withdrawal of Dox and that renewed proliferation is the direct result of $M y c / N I C D$ co-activation (Supplementary Fig. 7a-e).

Adult SCs and IHCs do not change their cell identities when dividing, suggesting limited reprogramming, which can be shown by re-expression of progenitor but not embryonic stem cell genes.
By qRT-PCR of cultured adult Dox-treated rtTA/tet-Myc/tetNICD cochleae (Supplementary Fig. 7a), we were able to detect the re-expression of inner ear progenitor genes including Six1, Eya1, Gata3 and Isl1 (Supplementary Fig. 7f). These genes are downstream of the Notch pathway and are involved in the formation of the auditory sensory epithelium ${ }^{34,36}$. Stem cell genes such as Nanog and Fut4 (Ssea1) were not upregulated. Cdkn1b (p27Kip1), a negative cell cycle regulator in the $\mathrm{SCs}^{12}$, was significantly down-regulated, supporting that MYC/NICD activities suppress $C d k n 1 b$ expression, which is, in part, involved in adult SC proliferation. The data support the idea that there is limited reprogramming by MYC/NICD-driven reactivation of inner ear progenitor genes.

To test the hypothesis that transient reprogramming is necessary for adult SC-to-HC transdifferentiation, we reversibly activated $M y c / N I C D$ in vivo by placing Dox-soaked gelfoam $(50 \mathrm{mg} / \mathrm{ml})$ in the round window niche of adult rtTA/tet-Myc/tetNICD cochleae for four days followed by ad-Atoh1 injection via cochleostomy (Fig. 4a). Two weeks after ad-Atoh1 injection, control adult rtTA/tet- $M y c /$ tet-NICD mice without Dox exposure had no new HCs (Fig. 4b). In Dox-treated rtTA/tet-Myc/tetNICD cochlea followed by ad-Atoh1 infection, we found ectopic HC-like cells (eHCs, defined as regenerated HC-like cells), labeled with HC markers ESPN and PVALB, regenerated in different cochlear areas (Fig. 4c) including the IHC region (Fig. 4d-f). Overall, the additional number of regenerated HC-like cells resulted in a cumulative increase in total $\mathrm{HC}$ number in comparison to the controls (Fig. 4i, j). Some of the eHCs were $\mathrm{EdU}^{+}$indicating regenerated HC-like cells were derived from dividing SC transdifferentiation (Fig. 4f, h). A large number of ectopic $\mathrm{HC}$-like cells $\left(\mathrm{ESPN}^{+}\right)$were regenerated in the limbus region (Fig. 4d, j; Supplementary Movie 1), the Claudius cell region (CldR) and the cochlear sensory epithelial region (SE) (Fig. 4i; Supplementary Fig. 8a, b). The majority of regenerated HC-like cells in the SE or CldR regions were from non-dividing $\left(\mathrm{EdU}^{-}\right)$cells (Supplementary Fig. 8a, b).

We further determined whether transient $M y c / N I C D$ activation is required for $\mathrm{HC}$ regeneration in adult mouse in vitro. Adding Dox to cultured adult rtTA/tet-Myc/tet-NICD cochleae for three days and following with ad-Atoh1 infection lasting for 14 days (Fig. 5a), we detected no new HCs in the Dox-only-treated control cochlea (Fig. 5b). In the ad-Atoh1-only-infected control cochlea, a small number of new HC-like cells were detected (Fig. 5c, e, f), all without induction of proliferation. In the adult rtTA/tet-Myc/tetNICD cochlea explant treated with Dox transiently followed by ad-Atoh1 infection, we detected robust $\mathrm{HC}$ regeneration $\left(\mathrm{MYO}^{+} / \mathrm{PVALB}^{+}\right)$in the sensory epithelial region (SE), the limbus regions (Lib) (Fig. 5d), and from transdifferentiation of both 
a

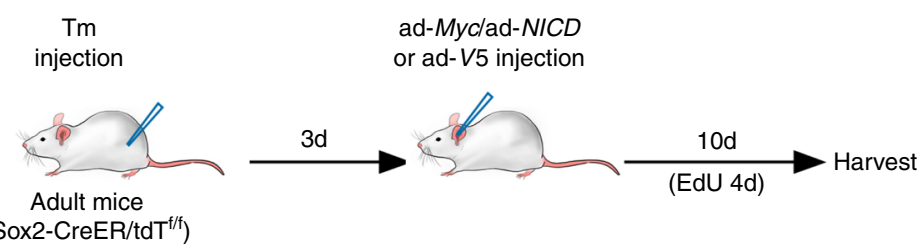

Sox2-tdT/MYO7A/EdU/DAPI

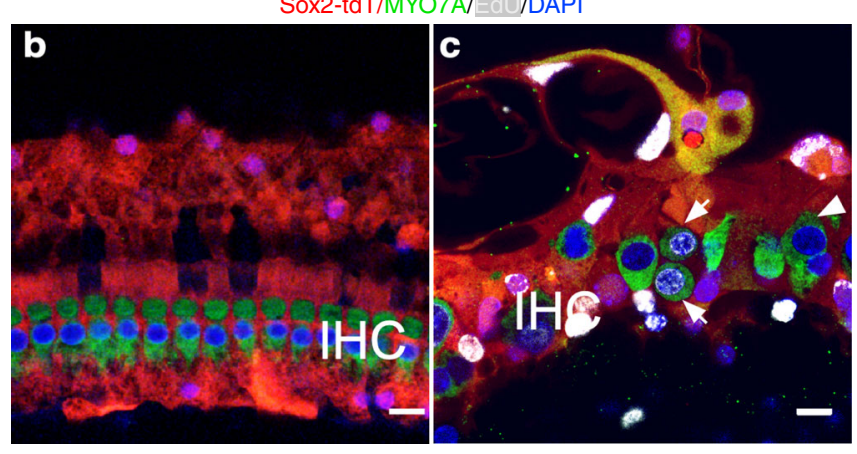

d

ad-Myc/ad-NICD/Tm

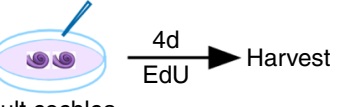

Adult cochlea

(Sox2-CreER/tdT ${ }^{\mathrm{f} / \mathrm{f}}$ )

tdT

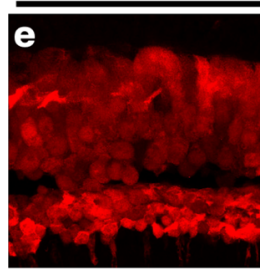

tdT

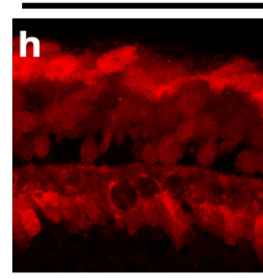

EdU

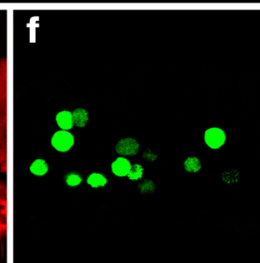

MKI67

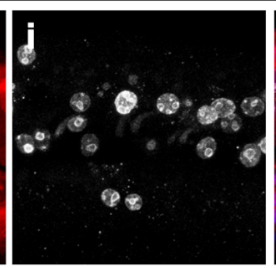

Merge/DAPI

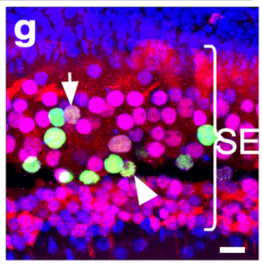

Merge/DAPI

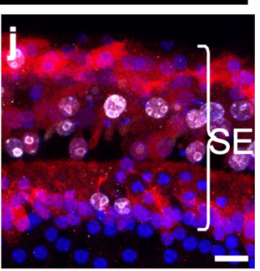

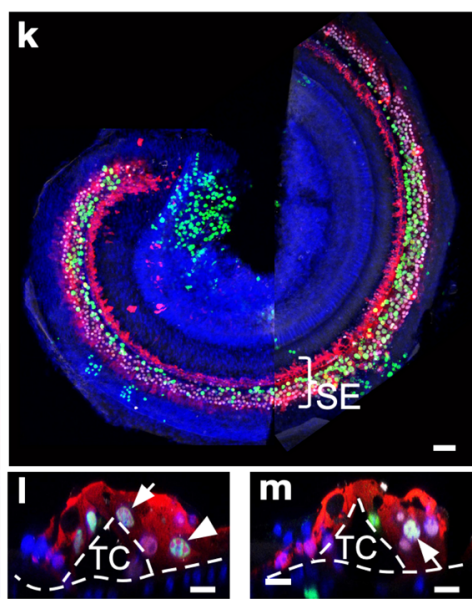

Fig. 3 Study of the origin of dividing HCs and SCs in adult cochlea by lineage tracing. a A schematic diagram depicting the in vivo experimental procedure. b In control adult Sox2-CreER/tdT/f mice 10 days after ad-V5 injection and Tamoxifen $(\mathrm{Tm})$ treatment, all IHCs were tdT negative and were surrounded by $\mathrm{tdT}+\mathrm{SCs}$. No EdU labeling was detected. c Following Tm treatment and ad-Myc/ad-NICD injection into adult Sox2-CreER/tdT f/f mouse cochlea for 10 days, dividing IHCs were detected (arrows). All IHCs (dividing, arrows; non-dividing, arrowhead) were tdT negative. $\mathbf{d}$ Schematic diagram of lineage-tracing of proliferating adult SCs in vitro. e-g EdU labeling in the SCs (tdT ${ }^{+}$) in cultured adult Sox2-CreER/tdT $\mathrm{T}^{\mathrm{f} f}$ cochlea after treatment with Tm and infection with ad-Myc/ad-NICD. Judging by their locations, likely dividing Deiters' cells (arrow) and pillar cells (arrowhead) were identified. Bracket indicates the sensory epithelium (SE). h-j MKI67 labeling in the SCs $\left(\mathrm{tdT}^{+}\right)$in cultured adult Sox2-CreER/tdT/f cochlea after treatment with Tm and infection with ad-Myc/adNICD. $\mathbf{k}$ A confocal image of the surface view of the middle turn of adult Sox2-CreER/tdT/f cochlea after treatment with Tm and infection by ad-Myc/adNICD to show numerous proliferating SCs $\left(\mathrm{tdT}^{+} / \mathrm{EdU}^{+}\right)$in the $\mathrm{SE}$ region. $\mathbf{I}, \mathbf{m}$ z-axis cross-section views of confocal images in (k) showed dividing SCs by triple labeling of $\mathrm{tdT}^{+} / \mathrm{EdU}^{+} / \mathrm{SOX} 2^{+}$(likely Deiters' cells, arrows; Hensen cell, arrowhead. tdT: red; EdU: green; SOX2: white). Dashed lines mark the basilar membrane and the tunnel of Corti (TC). Scale bars: $50 \mu \mathrm{m}$ in (k); $10 \mu \mathrm{m}$ in other figures.

dividing and non-dividing $\mathrm{SCs}\left(\mathrm{MYO}^{+} \mathrm{A}^{+} / \mathrm{SOX}^{+} / \mathrm{EdU}^{+}\right.$and $\mathrm{MYO}^{+} \mathrm{A}^{+} / \mathrm{SOX2}^{+} / \mathrm{EdU}^{-}$) (Arrows and arrowheads in Fig. $5 \mathrm{~d}$ ), respectively. Significantly more HC-like cells were regenerated after transient Myc/NICD activation followed by Atoh1 induction than Atoh1 induction alone (Fig. 5e, f). The cochlear regions in which HC-like cells were regenerated including the sensory epithelial region, the limbus region, and the Claudius cell region, were consistent between in vivo and in vitro studies. We, therefore, conclude that Myc/NICD co-activation successfully reprograms SCs, and their subsequent downregulation enables reprogrammed SCs (dividing and non-dividing) to then transdifferentiate into HC-like cells in response to Atohl for both in vivo and in vitro.

We hypothesized that sustained MYC/NICD activities leads to increase in apoptosis (Supplementary Fig. 5a-f). Conversely, transient MYC/NICD activities should lead to improved cell survival. To explore this idea, we performed study to compare cell 
a

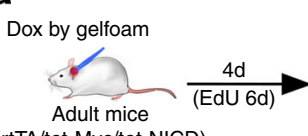

(rtTA/tet-Myc/tet-NICD)

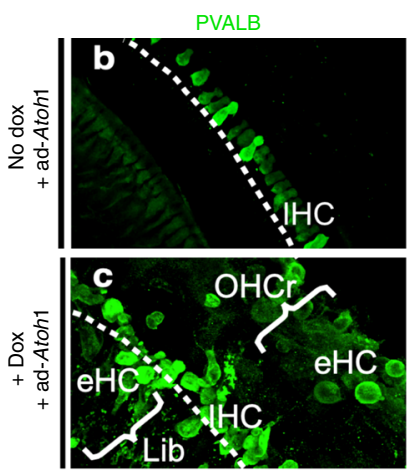

EdU
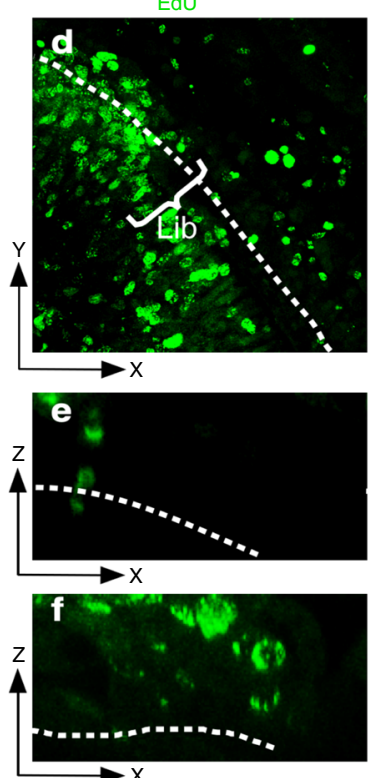

g

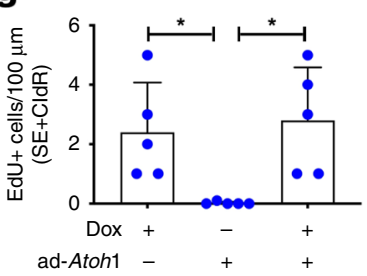

i

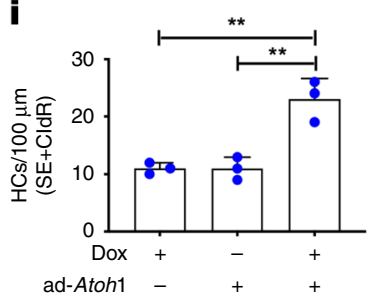

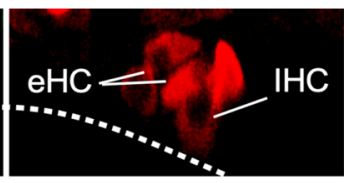
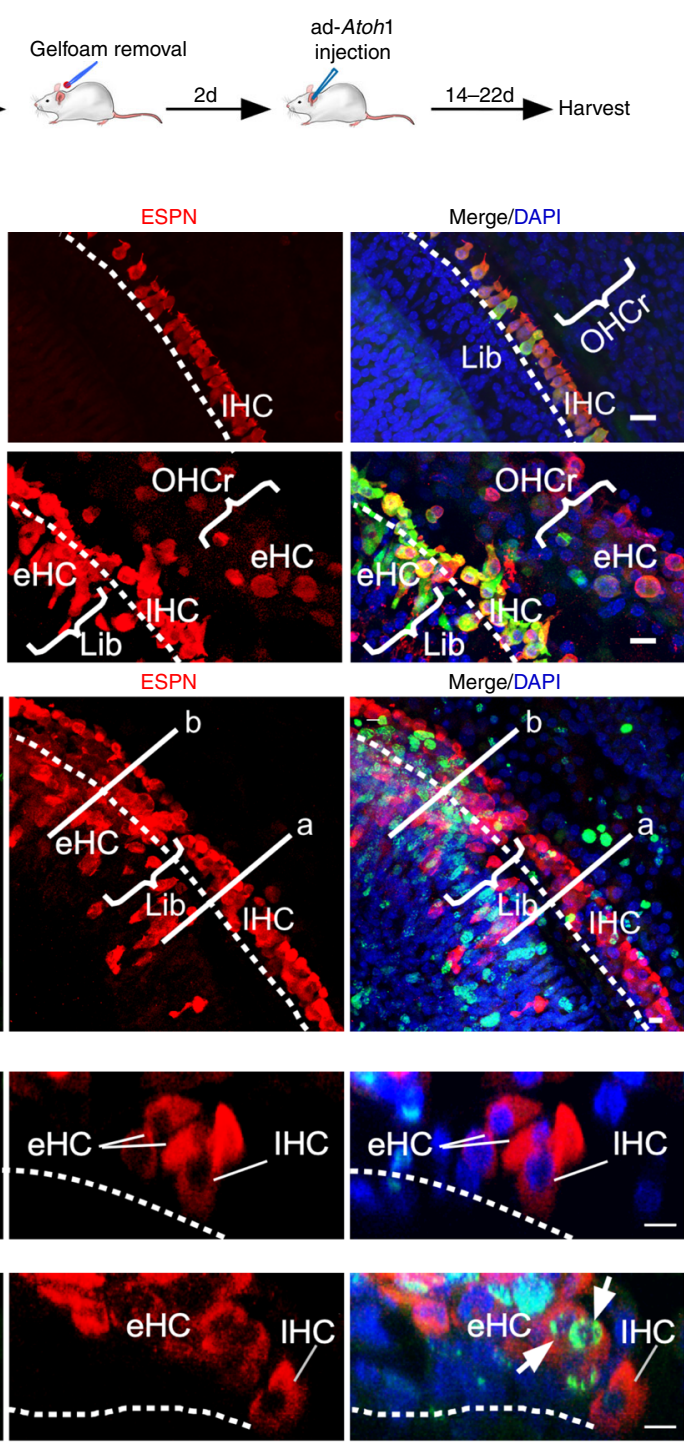

ESPN

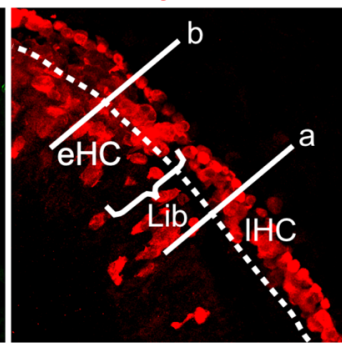

Merge/DAPI
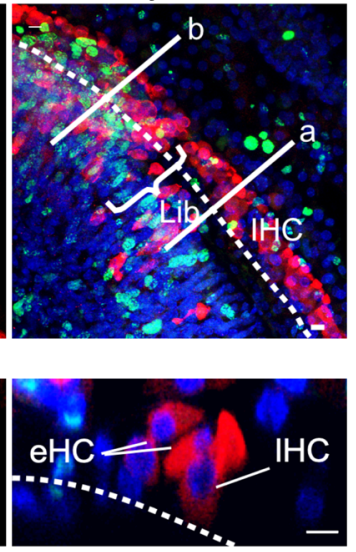

h

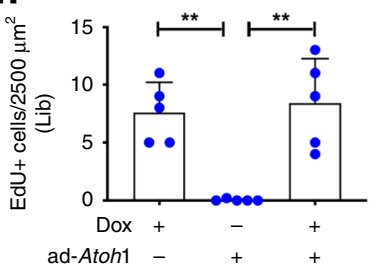

j

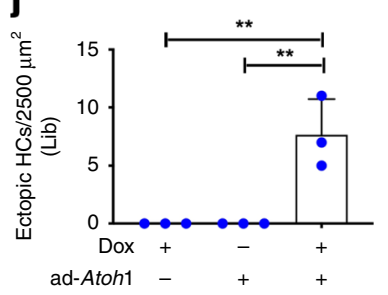

death index in cultured adult rtTA/tet-Myc/tet-NICD cochleae exposed to Dox for 7 or 14 days, respectively, with a total culture time of 14 days (Supplementary Fig. 5m). By CASP3 labeling, we found that cochleae exposed to Dox for 7 days had significantly fewer $\mathrm{CASP}^{+}$cells in the sensory epithelial region and a majority of SCs appeared healthy (Supplementary Fig. 5g-i, n). In contrast, the cochleae exposed to Dox for 14 days had a dramatic increase in the number of $\mathrm{CASP}^{+}$cells in the sensory epithelial region and the limbus region (Supplementary Fig. 5j-l,n). These results strongly support that transient $M y c / N I C D$ expression improves survival in reprogrammed SCs in adult cochlea, whereas continuous overexpression of $M y c / N I C D$ is detrimental to SC 
Fig. 4 Transient Myc/NICD activation is necessary for Atoh1-induced transdifferentiation of HC-like cells in vivo. a A schematic diagram illustrating the experimental procedure of transient Dox induction of Myc/NICD and HC induction by ad-Atoh1 in vivo. $\mathbf{b}$ In control adult (P30) rtTA/tet-Myc/tet-NICD mice injected with ad-Atoh 1 by cochleostomy for 20 days in vivo, existing IHCs were co-labeled with ESPN and PVALB, whereas all OHCs died because of the procedure in the mid-base turns. No new HCs were regenerated. Dashed line demarcated the boundary between IHCs and the limbus region (Lib). Bracket indicated $\mathrm{OHC}$ region $(\mathrm{OHCr}$ ). c In adult rtTA/tet-Myc/tet-NICD mice treated with Dox for 4 days followed by ad-Atoh1 infection for 22 days, new ectopic $\mathrm{HC}$-like cells (PVALB $+/ \mathrm{ESPN}^{+}$) were detected in the $\mathrm{OHC}$ region (bracket, $\mathrm{OHCr}$ ) and IHC region (eHC, bracket). $\mathbf{d}$ Upon gelfoam-mediated Dox induction to activate Myc/NICD for 4 days and ad-Atoh1 infection in adult rtTA/tet-Myc/tet-NICD mice in vivo for 14 days, SCs and non-SCs re-entered cell cycle $\left(\mathrm{EdU}^{+}\right)$and transdifferentiated to $\mathrm{HC}^{-l i k e}$ cells $\left(\mathrm{ESPN}{ }^{+}\right)$. The existing $\mathrm{IHCs}$ were indicated $(\mathrm{IHC})$, and the dashed line separated the IHC region from the limbus region (Lib). e A cross section view along the line (a) in (d), to illustrate an IHC and regenerated eHCs from SC transdifferentiation without proliferation. $\mathbf{f} A$ cross section view along the line (b) in (d), to show numerous eHCs (ESP ${ }^{+}$) regenerated in the limbus region, some of which were labeled with EdU (ESPN ${ }^{+} / \mathrm{EdU}^{+}$, arrows). The dashed lines demarcated the basilar membrane in (e, $\left.\mathbf{f}\right)$. $\mathbf{g}$-j Quantification in the mid-base turn showed significant increases in the numbers of EdU ${ }^{+}$cells $(\mathbf{g}-\mathbf{h})$, total HCs in the SE + CldR (i) and ectopic HC-like cells in Lib regions (j) in the experimental groups around the injection sites after Dox treatment and ad-Atoh1 infection, compared to the ad-Atoh1-injected control adult rtTA/tet-Myc/tet-NICD inner ear. ${ }^{\star} p<0.05$, ${ }^{\star \star} p<0.01$, one-way ANOVA with Tukey's multiple comparison test. Error bar, mean \pm s.d.; $(\mathbf{g}, \mathbf{h}): n=5 ;(\mathbf{i}, \mathbf{j}): n=3$. $n$ is the number of biologically independent samples. Scale bars: $10 \mu \mathrm{m}$. Source data are provided as a Source Data file.

survival. Transient $M y c / N I C D$ overexpression is thus essential to the survival of reprogrammed SCs as well as their capacity to transdifferentiate into HC-like cells.

Characteristics of new HC-like cells in adult cochlea. Auditory HCs can be divided into two specialized groups with distinct functions: OHCs and IHCs. Cochlear OHCs can be characterized by their anatomic position, electromotility properties, and their expression of OHC marker prestin (SLC26A5) ${ }^{35}$. We performed SLC26A5 labeling in rtTA/tet-Myc/tet-NICD cochleae after Dox/ ad-Atoh1 treatment (Fig. 6a) and detected SLC26A5 in some regenerated $\mathrm{HC}$-like cells $(28 \pm 13 \%$, mean \pm s.e.m., $n=5$ biologically independent samples) in the $\mathrm{OHC}$ region (Fig. 6b-e), indicating that a portion of regenerated HC-like cells in the $\mathrm{OHC}$ region start to express $\mathrm{OHC}$ markers.

What are the characteristics of regenerated HC-like cells? We hypothesized that new HC-like cells transdifferentiated from adult SCs may still have characteristics resembling developmentally young HCs, while those regenerated from adult IHCs may have a more mature differentiated status given their mature $\mathrm{HC}$ origin. To test this hypothesis, we used acetylated tubulin (AcTUBA4A) labeling to examine the presence of kinocilia, a structure only associated with young but not adult auditory HCs (Supplementary Fig. 9a) ${ }^{37}$. We found Ac-TUBA4A ${ }^{+}$HC-like cells in the OHC region in the Dox/ad-Atoh1-treated rtTA/tet$M y c /$ tet-NICD cochlea explant culture (Fig. 6f-h). PTPRQ, a marker for young IHCs and OHCs only evident in young mice up to 14 days postnatal (Supplementary Fig. $9 \mathrm{~b}$ ) ${ }^{38}$, was detected in regenerated HC-like cells in adult cochlea (Fig. 6j-m). In contrast, $\mathrm{HC}$-like cells regenerated from the division of existing $\mathrm{HCs}$ did not possess kinocilia (Fig. 6i). These results provide evidence to support "young" HC status of regenerated HC-like cells from SC transdifferentiation.

The stereocilia structure of HC-like cells were detected by actin and ESPN labeling (Fig. 6n-q). Using scanning electron microscopy (SEM), we identified HC-like cells by the presence of kinocilia and immature concentric stereocilia. In normal cochlea, $\mathrm{OHC}$ stereocilia are organized into a recognizable $\mathrm{V}$-formation (arrow, Fig. 6u) while IHC stereocilia are aligned across the cell's apical surface (arrowhead, Fig. 6u; Supplementary Fig. 10i). In contrast, immature stereocilia in regenerated HC-like cells are consistently short, either uniform in height, or disorganized (Fig. 6n-q, r-t; Supplementary Fig. 10a-h). In the control adult rtTA/tet-Myc/tet-NICD cochlea explant culture treated with Dox only or infected by ad-Atoh1 alone, no new HC-like cells were detected by SEM (Supplementary Fig. 10j-l). Regenerated HC-like cells were able to survive in culture for two weeks, reminiscent of neonatal auditory HCs that can survive in culture for a similar timeframe. In contrast, adult cochlear HCs usually die within $24 \mathrm{~h}$ in culture.
To study if regenerated HC-like cells in adult cochlea have functional transduction channels, we performed an FM1-43 uptake experiment. This fluorescent dye, and its fixable analog FM1-43FX, pass through functional transduction channels and are trapped by their charge within $\mathrm{HCs}^{39}$. Regenerated HC-like cells in vitro, from dividing and non-dividing SCs 14 days post infection, were able to take up FM1-43FX, whereas no such uptake was detected in the surrounding cells (Fig. 6v-y).

It is essential that regenerated HC-like cells are able to make connections with surrounding auditory neurons in order to form synapses that enable hearing. We used antibodies to the neurite marker neurofilament (NEFH) to stain adult rtTA/tet-Myc/tetNICD cochlea with regenerated HC-like cells after treatment with Dox/ad-Atoh1 in vivo. We found extensive neurite outgrowth to the sensory epithelial region, with the neurites wrapping around new HC-like cells. In contrast, in control cochleae without HC regeneration, virtually all the neurites retracted with few in contact with the existing IHCs (Supplementary Fig. 11a-e; Supplementary Movie 2).

mTOR is involved in $M y c / N I C D$ reprogramming in adult cochlea. Understanding the MYC/NICD-mediated pathway will shed light on the reprogramming mechanism and provide opportunities to fine-tune the process for improved regeneration efficiency. MYC and NOTCH have been shown to interact with the mTOR signaling pathway ${ }^{40-42}$. We hypothesized that the reprogramming effect in adult cochlea by MYC/NICD may be mediated in part by the mTOR pathway. To test the hypothesis, we examined production of phospho-rpS6 (p-rpS6), an mTOR effector, in Dox-treated adult rtTA/tet-Myc/tet-NICD cochlea in culture (Fig. 7a). We detected prominent labeling of p-rpS6 in dividing SCs (SOX2 ${ }^{+} / \mathrm{EdU}^{+} / \mathrm{p}-\mathrm{rpS} 6^{+}$, arrows, Fig. $7 \mathrm{c}$ ), but not in freshly dissected adult rtTA/tet-Myc/tet-NICD cochlea (Fig. 7b). To determine the role of mTOR in MYC/NOTCH-mediated proliferation, we incubated Dox-treated adult rtTA/tet-Myc/tetNICD cochlea culture with rapamycin, a well-known mTOR inhibitor ${ }^{43}$. The p-rpS6 signal was virtually eliminated by rapamycin despite Dox treatment (Fig. 7d, e). Moreover, rapamycin treatment significantly reduced the number of $\mathrm{EdU}^{+}$cells induced by $M y c / N I C D$ co-activation (Fig. $7 \mathrm{~d}$, f). The data strongly support that mTOR is downstream of MYC/NICD and is required in MYC/NICD-mediated proliferation.

To assess if mTOR plays a role in $\mathrm{HC}$ regeneration in adult cochlea, we compared the number of regenerated HC-like cells in Dox-treated adult rtTA/tet-Myc/tet-NICD cochleae infected by ad-Atoh1, with or without rapamycin treatment (Fig. 7g). Rapamycin treatment significantly reduced the number of regenerated $\mathrm{HC}$-like cells in the sensory epithelium (SE) or the 
a

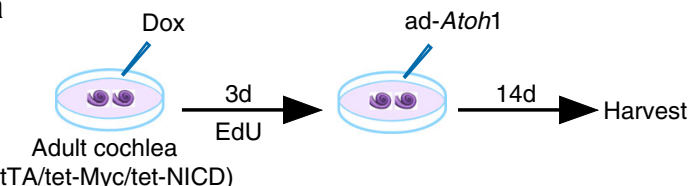

(rtTA/tet-Myc/tet-NICD)

EdU/MYO7A/PVALB/DAPI
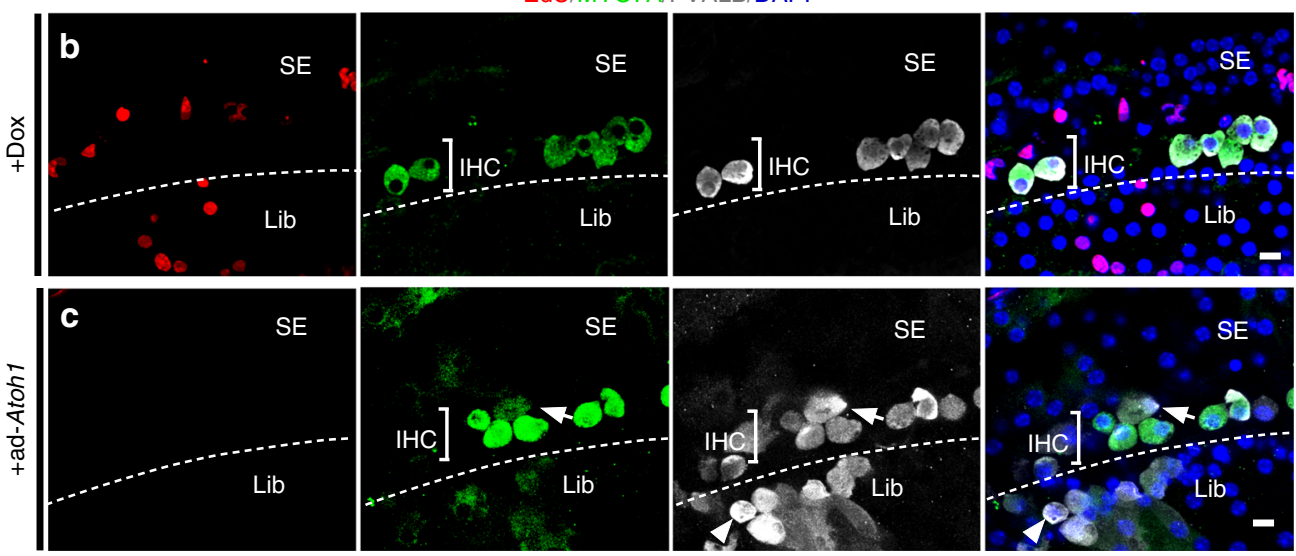

EdU/MYO7A/SOX2/DAPI
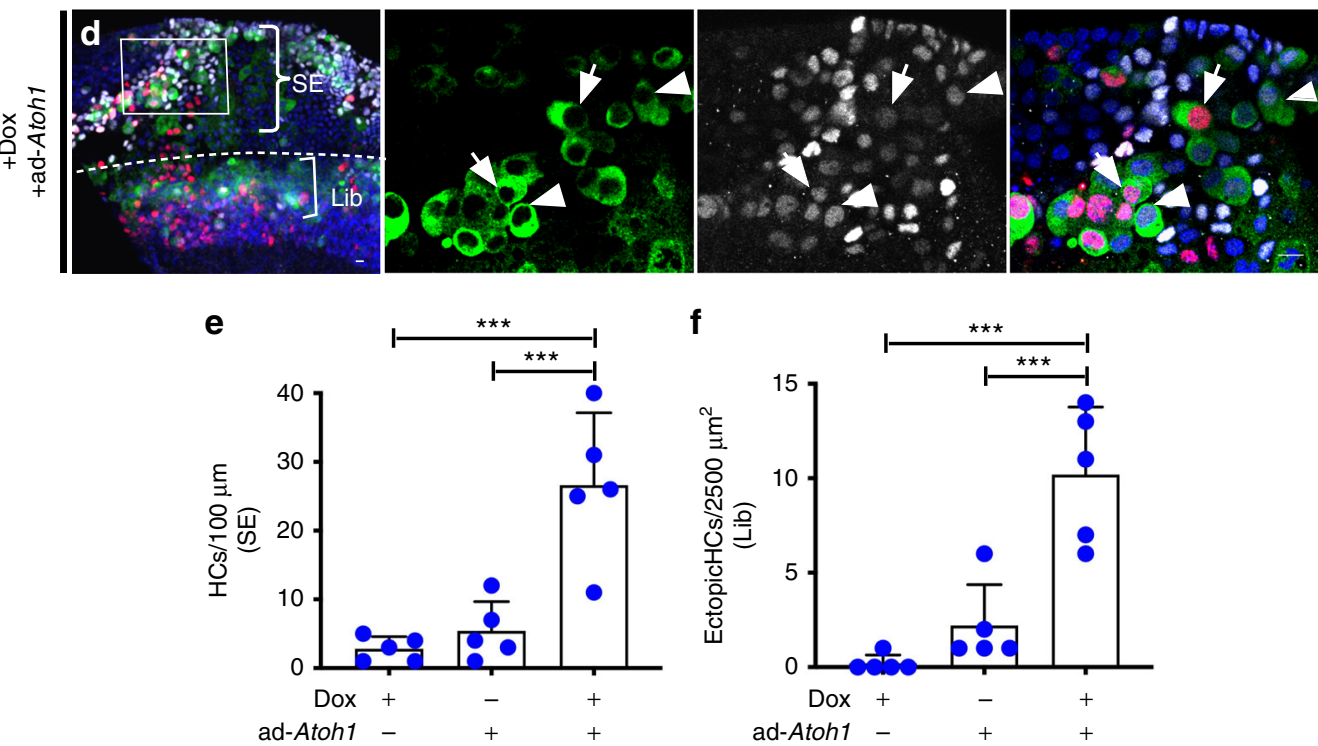

Fig. 5 Atoh7-induced regeneration of $\mathrm{HC}$-like cells through transient activation of Myc/NICD in vitro. a A schematic diagram illustrating in vitro transient Dox induction of Myc/NICD and HC induction by ad-Atoh1 in adult rtTA/tet-Myc/tet-NICD cochlea explant culture. b No new HCs were detected in the $\mathrm{OHC}$ region of the sensory epithelium (SE) in the cultured adult rtTA/tet-Myc/tet-NICD cochlea following 3 days in Dox and 14 days without Dox. Proliferating cells (EdU $\left.{ }^{+}\right)$and some surviving IHCs (MYO7A+/PVALB $\left.{ }^{+}\right)$were seen. $\mathbf{c}$ In the adult rtTA/tet-Myc/tet-NICD cochlea infected with ad-Atoh1 for 14 days, $\mathrm{PVALB}^{+}$cells in the limbus region (Lib) were detected. Weak MYO7A was detected in some PVALB ${ }^{+}$cells, with arrow and arrowhead pointing to such cells in the SE and Lib regions, respectively. $\mathbf{d}$ The mid-apex of the adult rtTA/tet-Myc/tet-NICD cochlea treated with Dox for 3 days followed by ad-Atoh1 transduction for 14 days. Left, a low-magnification image showed whole-mount adult rtTA/tet-Myc/tet-NICD cochlea treated by Dox and ad-Atoh1 with new HC-like cells from the sensory epithelium (SE, inset) and the limbus region (Lib). Middle to right, the enlarged inset showed new HC-like cells from dividing ( $\mathrm{MYO} \mathrm{A}^{+} / \mathrm{EdU}^{+}$, arrows) and non-dividing (MYO7A+/EdU-, arrowheads) SCs, all with SOX2 expression, indicating their SC origin. The dashed line demarcated the boundary between the SE and Lib regions in (b-d). e, $\mathbf{f}$ Quantification and comparison of regenerated HC-like cells (MYO7A ${ }^{+}$) in the apical turn of the cochlea culture. ${ }^{\star \star \star} p<0.001$, one-way ANOVA with Tukey's multiple comparison test. Error bar, mean $\pm \mathrm{s} . \mathrm{d} . ; n=5$ biologically independent samples for each group. Scale bars: Source data are provided as a Source Data file.

limbus regions (Fig. 7h-j), supporting that mTOR is further required for $\mathrm{HC}$ regeneration.

Given the prominent role that mTOR plays in proliferation and cell growth, we hypothesized that this pathway may compensate for part of MYC's functionality in adult inner ear reprogramming. To determine the extent by which mTOR activation is sufficient to replace MYC and induce proliferation in adult cochlea, we activated mTOR in Dox-treated rtTA/tet-
NICD adult cochlea culture by MHY1485, a small molecule mTOR activator ${ }^{44}$ (Fig. 7k). We observed EdU ${ }^{+}$cells in the SE and the limbus regions after MHY1485 treatment, but not in control rtTA/tet-NICD cochlea exposed to Dox alone (Fig. 7l-n). The number of EdU ${ }^{+}$cells was fewer after MHY1485 treatment and NICD activation compared to $M y c / N I C D$ co-activation (Fig. 7c), indicating that mTOR partially compensates for MYC function in renewed proliferation. We also observed more 
a

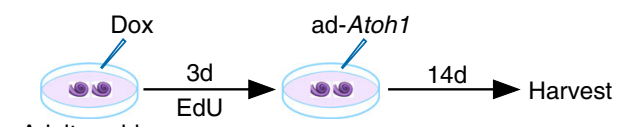

Adult cochlea (rtTA/tet-Myc/tet-NICD)

ESPN/SLC26A5/EdU/DAPI
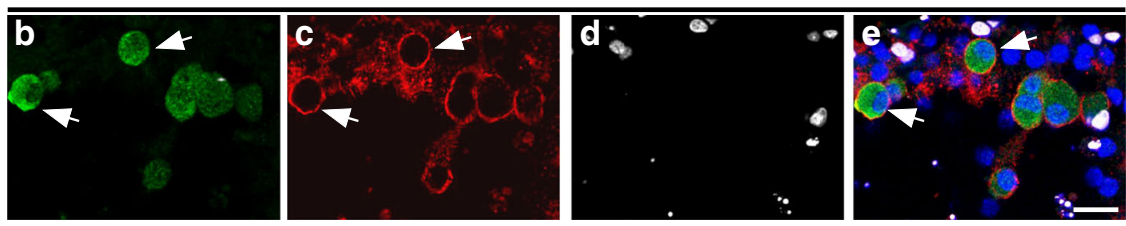

ESPN/Ac-TUBA4A/EdU/DAPI
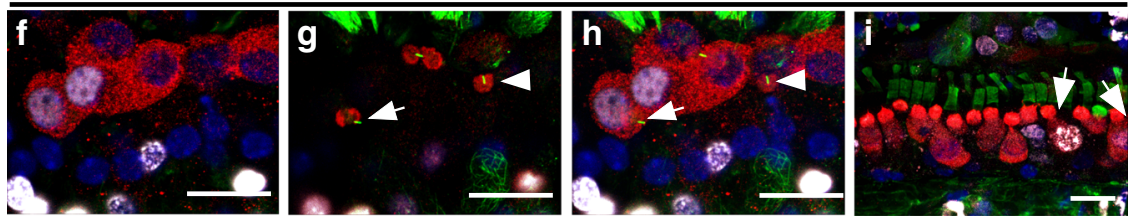

PTPRQ/PVALB/EdU/DAPI
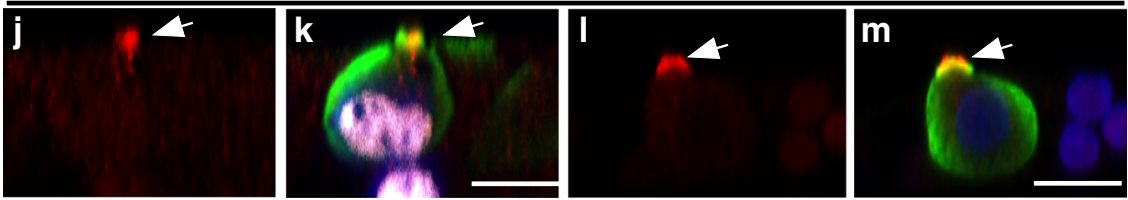

ACTB/PVALB
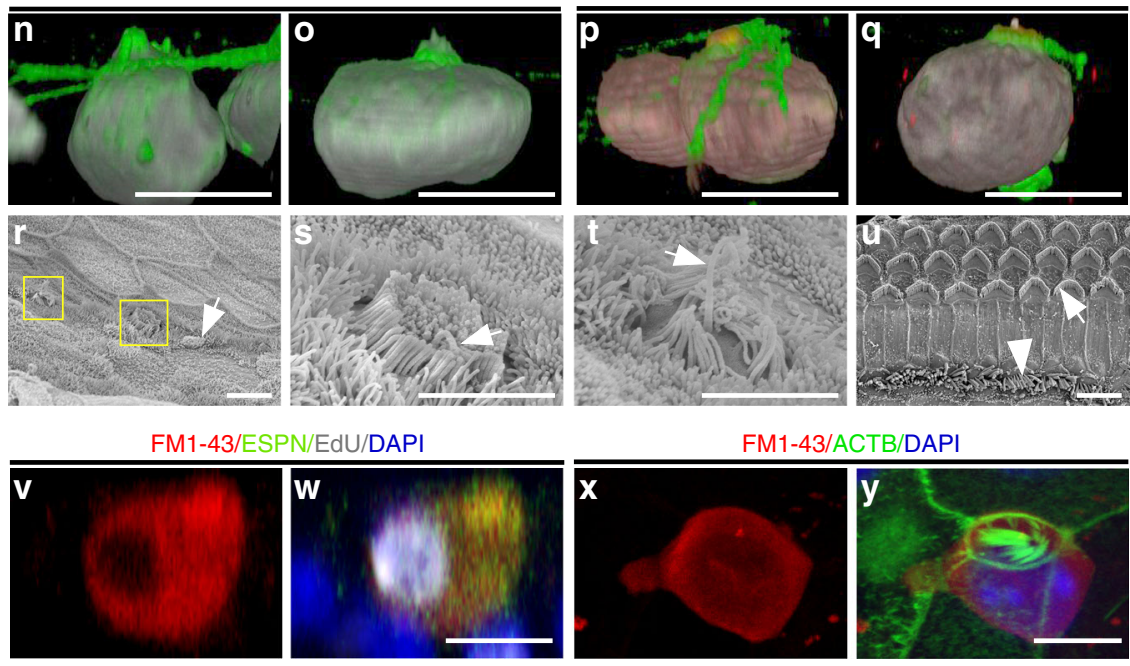

Fig. 6 Characteristics of regenerated HC-like cells. a A diagram illustrating the procedure of Dox and ad-Atoh1 treatment. b-e Membrane staining of SLC26A5 (prestin) was detected prominently in some regenerated $\mathrm{HC}$-like cells from SC transdifferentiation in the OHC region in vitro (arrows). f-i Kinocilia labeled with acetylated tubulin (Ac-TUBA4A) in rtTA/tet-Myc/tet-NICD cochlea treated with Dox and ad-Atoh1. The confocal images were from two different planes of the same sample ( $\mathbf{f}$ to show the nuclei; $\mathbf{g}$ to show the apical surface) with the merged image in (h). Kinocilia structure was detected in HC-like cells transdifferentiated from dividing (ESPN ${ }^{+} / \mathrm{EdU}^{+}$, arrow in $\mathbf{g}, \mathbf{h}$ ) and non-dividing (ESPN ${ }^{+} / \mathrm{EdU}^{-}$, arrowhead in $\mathbf{g}$, $\mathbf{h}$ ) SCs, respectively, but not detected in the dividing IHCs (ESPN +/EdU +, arrows in $\mathbf{i}$ ). $\mathbf{j}-\mathbf{m} 17$ days after Dox/ad-Atoh1 treatment of the cultured adult rtTA/tet-Myc/tet-NICD cochlea, PTPRQ (young OHC marker) was detected in the hair bundles of dividing (j, $\mathbf{k}$, arrow) and non-dividing (I, $\mathbf{m}$, arrow) HC-like cells. $\mathbf{n}$-q 3D-reconstruction of regenerated HC-like cells $\left(\mathrm{ESPN}^{+} / \mathrm{PVALB}^{+}\right)$in the $\mathrm{OHC}$ region. Different shapes of hair bundles can be seen (labeled with phalloidin, green). $\mathbf{r}$ Images of scanning electron microscopy (SEM) showing immature stereocilia from new HC-like cells in rtTA/tet-Myc/tet-NICD cochlea treated with Dox and ad-Atoh1 in vitro (squares and arrow). $\mathbf{s ,} \mathbf{t}$ Enlarged images from squares in $6 r$ to show short and uniform height stereocilia with a kinocilium (arrow in $\mathbf{s}$ ) and disorganized stereocilia with a kinocilium (arrow in $\mathbf{t}$ ). $\mathbf{u}$ An image of SEM of adult HC stereocilia in freshly dissected cochlea from P30 rtTA/tet-Myc/tet-NICD mouse with typical stereocilia in V-shape in OHCs and linear alignment in IHCs. v-y 17 days after Dox/ad-Atoh1 treatment of the cultured adult rtTA/tet-Myc/tet-NICD cochlea, regenerated HC-like cells $\left(\mathrm{ESPN}^{+}\right.$or $\left.\mathrm{ACTB}^{+}\right)$, transdifferentiated either from dividing SC $(\mathbf{w})$ or non-dividing $\mathrm{SC}(\mathbf{y})$, were shown to take up FM1-43FX. Other surrounding non-HC cells $\left(\mathrm{DAPI}^{+} / \mathrm{ESPN}^{-}\right)$did not take up FM1-43FX (w). ACTB: actin. Scale bars: $2 \mu \mathrm{m}$ in $\mathbf{r}-\mathbf{u} ; 10 \mu \mathrm{m}$ in other figures.

HC-like cells after Dox-induced NOTCH activation, MHY1485 treatment together with ad-Atoh1 infection than by Dox-induced NOTCH activation and ad-Atoh1 infection in cultured rtTA/tetNICD adult cochlea (Fig. 7l, m, o), suggesting that MHY1485 and
NICD work synergistically to promote HC regeneration. We conclude that the reprogramming effect by $M y c / N I C D$ in proliferation and $\mathrm{HC}$ regeneration is partially mediated by the mTOR signaling pathway. 
a

Dox+Rapamycin

Adult cochlea

(rtTA/tet-Myc/tet-NICD)

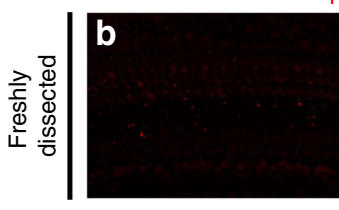

P-rpS6/PVALB/SOX2/DAPI
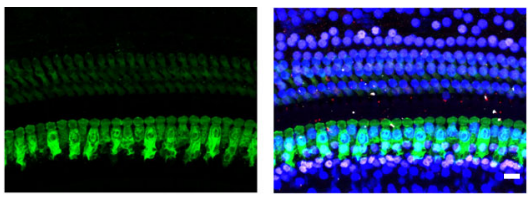

p-rpS6/EdU/SOX2/DAP
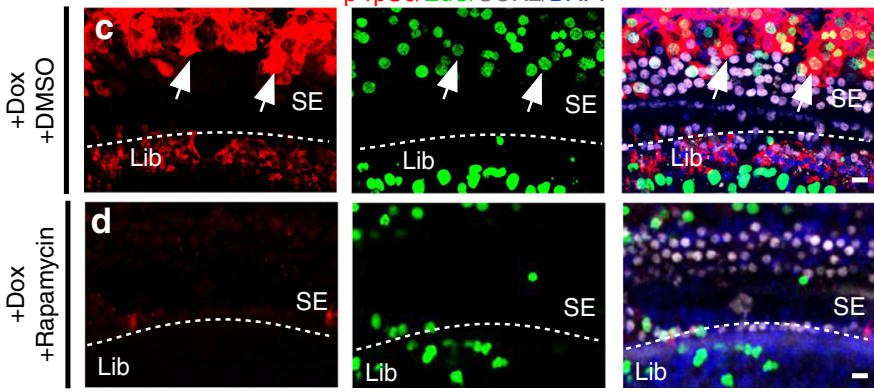

g

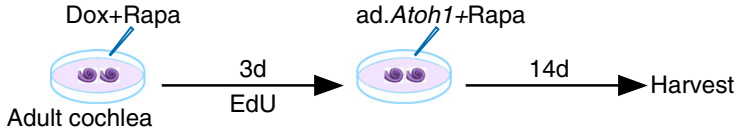

(rtTA/tet-Myc/tet-NICD)
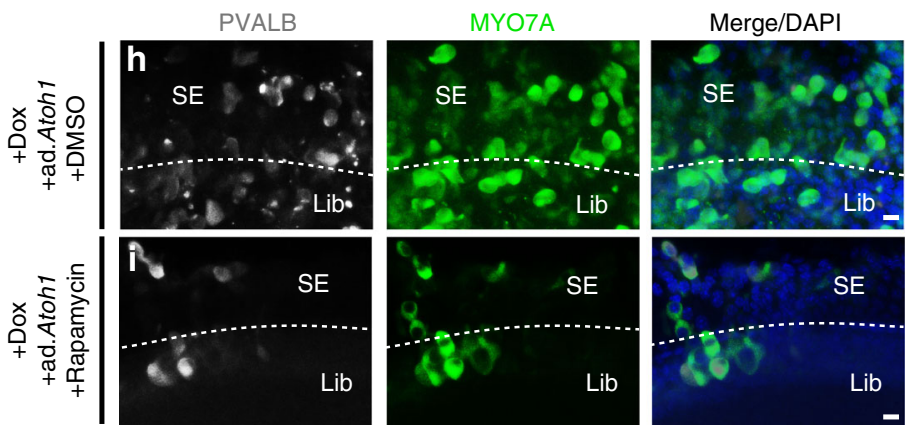

k

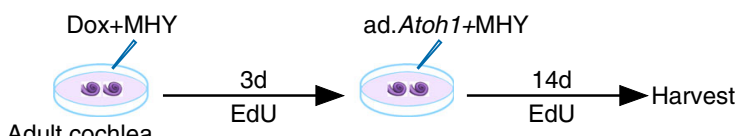
(rtTA/tet-1
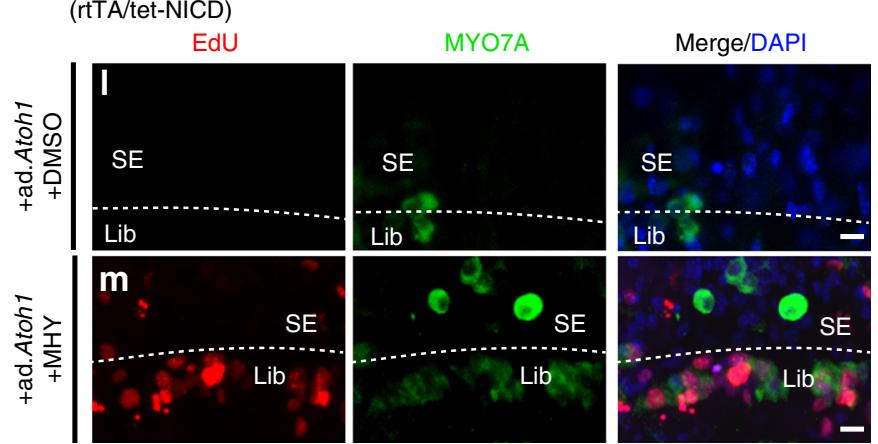
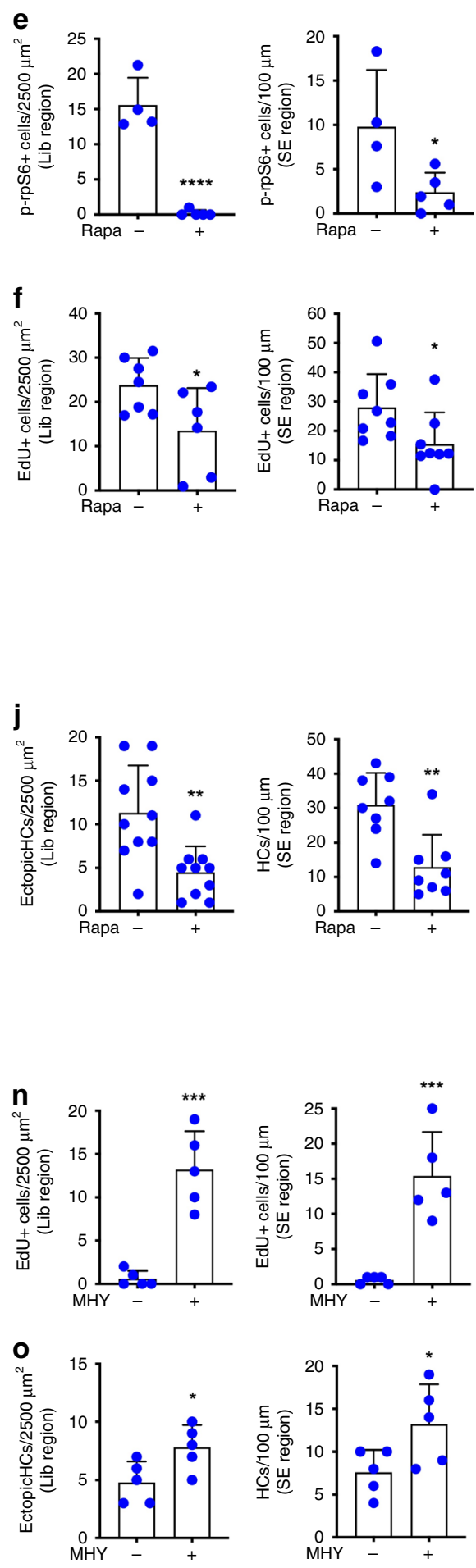

\section{Discussion}

Each of the diverse inner ear cell types, including HCs, SCs, neurons and the cells within the stria vascularis, play an essential role in hearing ${ }^{45}$. The lack of the capacity to proliferate in the damaged adult inner ear has been a major hurdle to regenerate any of the cell types, which ultimately leads to permanent hearing loss. In human newborns, the inner ear is fully mature. Therefore, regeneration of inner ear cells in adult by renewed proliferation and/or transdifferentiation is an essential step towards the development of new treatments for hearing restoration.

By combined activation of $M y c$ and Notch1, we demonstrate that multiple adult cochlear cell types can be induced to re-enter 
Fig. 7 mTOR is downstream of the MYC/NICD pathway. a A diagram illustrating the procedure of Dox and rapamycin treatment on adult rtTA/tet-Myc/ tet-NICD cochlea in vitro. b Phospho-rpS6 (p-rpS6), a downstream effector of mTOR signaling, was not detected in freshly dissected adult cochlea. c Doxinduced Myc/NICD activation upregulated p-rpS6 in cultured adult rtTA/tet-Myc/tet-NICD cochlea. Arrows pointed to the dividing SCs labeled with p-rpS6. d 6 days after Dox and rapamycin co-treatment of cultured adult rtTA/tet-Myc/tet-NICD cochlea, p-rpS6 was greatly reduced and the numbers of $\mathrm{EdU}^{+}$and $\mathrm{p}-\mathrm{rpS6} 6^{+}$cells decreased significantly in the sensory epithelial region (SE) and limbus region (Lib) compared to Dox-treated cochlea (c). e, f Quantification of $\mathrm{p}-\mathrm{rpS6}^{+}$and EdU ${ }^{+}$cells in the cultured adult rtTA/tet-Myc/tet-NICD cochleae exposed to DOX, with or without rapamycin treatment. ${ }^{\star} p<0.05,{ }^{\star \star \star \star} p<0.0001$, two-tailed unpaired Student's $t$-test. Error bar, mean \pm s.d.; e: $n=5$ for rapamycin-treated groups and $n=4$ for controls. f: Lib region, $n=6$ for rapamycin-treated group and $n=7$ for control group; SE region, $n=8$ for both groups. $\mathbf{g}$ A diagram illustrating the study of rapamycin treatment on $\mathrm{HC}$ induction by Atoh1. $\mathbf{h}, \mathbf{i}$ The numbers of EdU + cells and new $\mathrm{HC}$-like cells in the $\mathrm{SE}$ and Lib were significantly decreased in the rapamycintreated adult rtTA/tet-Myc/tet-NICD cochleae following 3-day Dox treatment and ad-Atoh1 infection for 14 days. $\mathbf{j}$ Quantification of total HCs and ectopic $\mathrm{HC}$-like cells $\left(\mathrm{MYO}^{+}\right)$in the apex turn of cochleae in $(\mathbf{h}, \mathbf{i}) .{ }^{\star \star} p<0.01$, two-tailed unpaired Student's $t$-test. Error bar, mean $\pm \mathrm{s}$.d.; Lib region, $n=10$ for both groups; SE region, $n=8$ for both groups. $\mathbf{k}$ A diagram illustrating the study of the effect of MHY1485 on proliferation and HC regeneration.

I, $\mathbf{m}$ Significantly more EdU + cells and new HC-like cells in SE and Lib in the apex turn were observed in the MHY1485-treated adult rtTA/tet-NICD cochlea following 3-day Dox treatment and ad-Atoh1 infection for 14 days, compared to DMSO-treated controls. n, o Quantification of EdU ${ }^{+}$cells, MYO7A ${ }^{+} \mathrm{HCs}$ and ectopic HC-like cells in $(\mathbf{I}, \mathbf{m}) .{ }^{*} p<0.05,{ }^{* \star *} p<0.001$, two-tailed unpaired Student's t-test. Error bar, mean $\pm s . d . ; n=5$ for each group. $n$ is the number of biologically independent samples. Scale bars: $10 \mu \mathrm{m}$. Source data are provided as a Source Data file.

and complete the cell cycle with dividing cochlear cells maintaining their identities. By transiently modulating MYC/NICD activities, adult cochlear SCs are reprogrammed and can respond to the induction signal by Atoh1 to transdifferentiate into HC-like cells efficiently in vivo and in vitro. This work forms the basis for our continued study of hearing recovery in adult deaf animal models.

Efforts towards inner ear regeneration have been centered around HCs using a myriad of approaches including Atoh1 overexpression, inner ear stem cells, induction of proliferation in the cochlea, and, recently, direct conversion of SCs to HCs by multiple transcription factors ${ }^{11,17-19,46,47}$. Because SCs and remaining $\mathrm{HCs}$ are already in their proper locations in the cochlea, renewed proliferation and $\mathrm{HC}$ regeneration in situ would be ideal for function restoration.

$M y c$, a cell cycle activator and a universal amplifier of expressed genes ${ }^{30}$, is not involved in normal inner ear development $^{48}$. Myc overexpression induces proliferation in adult mouse utricular $\mathrm{SCs}^{49}$ and in adult retinal axon regeneration ${ }^{50}$. In zebrafish neuromasts, $m y c$ is rapidly activated upon $\mathrm{HC}$ damage and is required for SC proliferation and $\mathrm{HC}$ regeneration ${ }^{23}$, supporting the critical role that transient $M y c$ activation in renewed proliferation is necessary for $\mathrm{HC}$ regeneration. It is significant that terminally differentiated adult cochlear cells are capable of cell cycle re-entry without undergoing drastic de-differentiation: proliferating IHCs regenerate relatively mature IHCs expressing HC marker ESPN (Supplementary Fig. 1i) and IHC marker SLC17A8 (VGLUT3) ${ }^{51}$ (Fig. 2n); whereas adult cochlear SC subtypes in cell division express SC markers (Supplementary Fig. 4). The maintenance of cell identity during proliferation is likely due to limited reprogramming, which is supported by upregulation of inner ear progenitor genes but not stem cell genes (Supplementary Fig. 7f). We have previously shown that Notch inhibition promotes the Wnt pathway, which promotes $\mathrm{Lgr5}^{+}$ cochlear progenitor cells to enter cell cycle and regenerate HCs in neonatal mice ${ }^{52}$. Compared to the current study, it is suggested that NOTCH may play a role in inner ear progenitors that is different from those in the adult cochlea in which Notch activation is required for proliferation of multiple SC subtypes, and renewed proliferation does not depend on the presence of progenitor cells.

Our study illustrates a two-stage process in cell cycle re-entry and subsequent $\mathrm{HC}$ regeneration. First, SCs are reprogrammed by $M y c / N I C D$ co-activation that is necessary to induce reprogramming and renewed proliferation. Subsequently, $M y c / N I C D$ needs to be down-regulated to allow reprogrammed SCs to respond to the HC induction signals (e.g. Atoh1 overexpression) to transdifferentiate into HC-like cells. Downregulation of $\mathrm{NOTCH}$ is necessary to $\mathrm{HC}$ regeneration likely because NOTCH activates the targets Hes 1 and Hes 5 which are both antagonists of Atoh $1^{35}$. For HC regeneration, Hes 1 and Hes 5 need to be silenced, which can be achieved by Notch downregulation and Atoh1 overexpression. Further, transient $M y c$ function is likely important in SC-to-HC transdifferentiation, as it is known that continuous overexpression of $M y c$ is detrimental to differentiation ${ }^{53}$.

In the rtTA/tet-Myc/tet-NICD mouse model in vivo, HC-like cells are regenerated from the IHC region almost exclusively by transdifferentiation of non-dividing SCs after reprogramming; whereas the HC-like cells are regenerated from transdifferentiation of dividing and non-dividing cells in the limbus region (Fig. 4d-f; Supplementary Fig. 8a, b). The data strongly supports that $M y c / N I C D$-mediated reprogramming, not proliferation per se, is a prerequisite to robust SC-to-HC transdifferentiation. HC-like cells can be regenerated from multiple regions within the cochlea including the limbus region and the Claudius cell region, in addition to the sensory epithelial region. Thus, multiple adult cochlear cell types have the potential to become HC-like cells. For hearing restoration, however, it is preferable that regeneration could be confined to the sensory epithelial region so that regenerated HCs may directly contact the tectorial membrane to elicit mechanical transduction. In addition to Atoh1 overexpression, our study points to an alternative approach of active blockade of Notch function following $M y c / N I C D$ co-activation by interrupting the binding of NOTCH and its partner RBPJ with inhibitors or suppressing Hes1/Hes5 by siRNAs, to regenerate HCs long after HC damage. These transitory approaches may enhance HC maturation and survival in comparison to continuous Atoh1 overexpression, which is incompatible with $\mathrm{HC}$ maturation ${ }^{54}$.

An understanding of the mechanism underlying the differences between transient and sustained $M y c / N I C D$ activation should shed light on SC reprogramming, renewed proliferation and improvement in $\mathrm{HC}$ regeneration efficiency. The mTOR pathway plays important roles in cell growth, survival and proliferation through regulation of multiple cell processes ${ }^{55,56}$. Up-regulating mTOR activity by pTEN deletion leads to constitutive neurogenesis in brain and regeneration of axons ${ }^{57,58}$. In the mammalian inner ear, the mTOR pathway is involved in proliferation of vestibular SCs that can be suppressed by the mTOR inhibitor rapamycin ${ }^{59}$. The discovery of the $\mathrm{mTOR}$ pathway as a major downstream component of Myc-Notch1 co-activation in the induction of proliferation and $\mathrm{HC}$ regeneration efficiency is consistent with the roles of mTOR. As mTOR is not active in normal adult cochlea (Fig. 7b), reactivation of the mTOR pathway together with Notch1 may provide another route to induce limited reprogramming and proliferation or HC regeneration. Such a combination may allow us to fine-tune the process of 
reprogramming related to proliferation and $\mathrm{HC}$ regeneration, so the number of cell cycles can be precisely controlled to produce a defined number of HCs and SCs for hearing recovery.

Well documented is the presence of undifferentiated flat epithelial cells instead of SCs in the cochlea of patients suffering from severe hearing loss ${ }^{60}$. The flat epithelium loses responsiveness to Atoh 1 and does not become $\mathrm{HCs}^{61}$. Our study points to the possibility of deeper reprogramming, which could be achieved by activation of the otic genes upstream of Notch during development with $M y c$ in the flat epithelium so these cells can first become SC-like and then transdifferentiate into HCs.

Regenerated HC-like cells are capable of promoting outgrowth of adult neurites, strongly supporting that new HCs play a dominant role in attracting adult neurites to re-establish HCneuron connections. Elucidation of the underlying mechanism may lead to strategies for recovery of the lost connections between HCs and neurons in hidden hearing loss ${ }^{62}$. Altogether, our study lays a foundation to study hearing recovery through regeneration of $\mathrm{HCs}$, SCs, and reconnection to neurons in the adult mammalian inner ear.

Reprogramming and proliferation induced by $M y c / N I C D$ coactivation in the adult cochlea is widespread, encompassing SCs, $\mathrm{HCs}$, and fibrocytes in the stria vascularis, providing a useful tool to regenerate diverse inner ear cell types involved in different types of deafness ${ }^{63,64}$. Given the important roles $M y c$ and Notch play in different tissues ${ }^{65-67}$, we hope that our study can serve as a model for regeneration of other post-mitotic tissues such as the retina and the central nervous system.

\section{Methods}

Mouse models. Rosa-NICD, Rosa-rtTA (rtTA) and tdTomato reporter (tdT) mice were from Jackson Laboratory (Stock\# 008159, 006965 and 007914); tet-on-Myc mice ${ }^{68}$ were from Dr. M. Bishop of the University of California, San Francisco; and tet-on-NICD mice ${ }^{69}$ were from Dr. D. Melton of Harvard University. For the transgenic mice, the background was mixed C57/129SvJ, with roughly equal numbers of sexes. The wild type mice were C57BL/6 from Charles River Laboratories.

Viral Injection in vivo. All adult mice used were between 6 weeks to 8 weeks old, except for those specifically mentioned. For viral injection, cochleostomy was performed on the anesthetized mice by opening the bulla, and adenovirus with a titer of $5 \times 10^{12} \mathrm{pfu} / \mathrm{ml}$ was injected into the scala media by a pressure-controlled motorized microinjector at the speed of $3 \mathrm{nl} / \mathrm{sec}$. A total of $0.32 \mu \mathrm{l}$ of adenovirus was injected into each cochlea. For viral mixtures, an equal volume was used for each virus. BrdU (Sigma) or EdU (Santa Cruz Biotech) was injected to the mice i.p. $16 \mathrm{~h}$ after viral injection at a concentration of $50 \mathrm{mg} / \mathrm{kg}$ daily for 4 or 6 days, with the injected animals harvested at various time points $(4,8,12,15$ and 35 days). The use of animals was approved by the Massachusetts Eye \& Ear Infirmary IACUC, with the treatment of animals following the approved protocols. The viral use was approved by the Harvard COMS committee.

Induction of $\mathbf{M y c / N I C D}$ in vivo. To induce $M y c / N I C D$ in vivo, otic bullas were opened to expose the round window niche. For transient Dox exposure, gelfoam sponge (Pfizer) soaked with Dox in DMSO at the concentration of $50 \mathrm{mg} / \mathrm{ml}$ was placed outside the round window niches for 4 days. The gelfoam was surgically removed 2 days before ad-Atohl infection.

Adult cochlear culture and viral infection in vitro. Different from the neonatal cochlea culture method, in which the cochleae were disassociated from the bone, adult mouse whole cochleae (6-8 weeks old) were dissected with the bone attached. The bulla was first removed from the skull, with the bulla briefly dipped in $70 \%$ ethanol before being placed in ice-cold HBSS. The vestibular region was also removed. Under a dissecting microscope, the middle ear, vessels and the debris were removed from the bulla. The bone covering the apical turn were removed, and round window and oval window membranes were opened to allow media exchange with the cochlea fluids. The ligament portion and Reissner's membrane at each end of the cochlea were also removed to facilitate the access of medium to the sensory epithelial region. The cochleae were maintained in floating culture in DMEM/F12 (Invitrogen) supplemented with N2 and B27 (both from Invitrogen) for 3-22 days. For infection, adenovirus was added to the culture at a titer of $5 \times 10^{10} \mathrm{pfu} / \mathrm{ml}$ overnight before the replacement with fresh medium. ad-Atoh 1 and ad-V5 were purchased from the SignaGen Laboratories, Rockville, MD. ad-NICD-V5 was a gift from Dr. Igor Prudovsky, Maine Medical Center Research Institute. ad-Cre-GFP and ad-GFP were purchased from the Vector Lab, Baylor College of Medicine, Houston. Ad-Myc has been previously described ${ }^{27}$. To label proliferating cells, $\mathrm{BrdU}$ and EdU were added at a final concentration of $10 \mu \mathrm{M}$ for varying time periods.

Induction of $\mathbf{M y c / N I C D}$ in vitro for HC regeneration. For in vitro studies, cultured 6-week-old Dox-inducible Rosa-rtTA/tet-Myc/tet-NICD cochleae were treated with Dox (Sigma, $2 \mu \mathrm{g} / \mathrm{ml}$ final concentration) for 3 days with EdU $(10 \mu \mathrm{M})$, followed by ad-Atoh $1\left(5 \times 10^{10} \mathrm{pfu} / \mathrm{ml}\right)$ infection overnight. The controls were cultured adult cochleae of the same genotype treated with Dox or infected with adAtoh1, respectively. The culture was replaced with fresh medium for additional 14 days. In the studies of the mTOR pathway, rapamycin (Sigma, $10 \mu \mathrm{M}$ final concentration) or MHY 1485 (Millipore, $20 \mu \mathrm{M}$ final concentration) were added to the culture with Dox. The cochleae were harvested and decalcified before immunohistochemistry.

Lineage tracing. For in vivo lineage tracing studies, 6-week-old Sox2-CreER/tdT $\mathrm{f} / \mathrm{f}$ mice were injected with tamoxifen (Sigma, $200 \mathrm{mg} / \mathrm{kg}$ ) daily for three days before injection of ad-Myc/ad-NICD $(0.3 \mu \mathrm{l}$ per injection) into the cochlea via cochleostomy. BrdU or EdU $(50 \mathrm{mg} / \mathrm{kg})$ was injected i.p. daily for 6 days. ad-V5injected mice were controls. Six to 12 days after ad-Myc/ad-NICD injection, the mice were sacrificed, and cochleae dissected for processing.

For in vitro lineage tracing studies with triple virus, 6-week-old Sox2-CreER/ $\operatorname{tdT}^{\mathrm{f} / \mathrm{f}}$ mice were injected with tamoxifen $(75 \mathrm{mg} / \mathrm{kg})$ daily for three days before the cochleae were dissected for culture. Triple virus mixture (ad-Myc/ad-NICD/adAtoh 1 ) was added to the medium for $16 \mathrm{~h}$ at a concentration of $10^{9} \mathrm{pfu} / \mathrm{ml}$ per virus. Fresh medium was subsequently used with $\mathrm{EdU}(10 \mu \mathrm{M})$ for culture for additional 14 days before harvest.

Reporting summary. Further information on research design is available in the Nature Research Reporting Summary linked to this article.

\section{Data availability}

The source data underlying Fig. 1n, o, 2h-k, q-t, 4g-j, 5e, f, 7e, f, j, n, o, Supplementary Figs. $1 \mathrm{~d}, 2 \mathrm{e}, \mathrm{f}, 4 \mathrm{~d}, \mathrm{~g}, 5 \mathrm{n}$, and $7 \mathrm{e}, \mathrm{f}$ are provided as a Source Data file.

Received: 14 November 2018; Accepted: 13 October 2019;

Published online: 04 December 2019

\section{References}

1. Corwin, J. T. \& Cotanche, D. A. Regeneration of sensory hair cells after acoustic trauma. Science 240, 1772-1774 (1988).

2. Ryals, B. M. \& Rubel, E. W. Hair cell regeneration after acoustic trauma in adult Coturnix quail. Science 240, 1774-1776 (1988).

3. Corwin, J. T. Postembryonic production and aging in inner ear hair cells in sharks. J. Comp. Neurol. 201, 541-553 (1981).

4. Jones, J. E. \& Corwin, J. T. Replacement of lateral line sensory organs during tail regeneration in salamanders: identification of progenitor cells and analysis of leukocyte activity. J. Neurosci. 13, 1022-1034 (1993).

5. Dooling, R. J., Ryals, B. M. \& Manabe, K. Recovery of hearing and vocal behavior after hair-cell regeneration. Proc. Natl Acad. Sci. USA 94, 14206-14210 (1997).

6. Warchol, M. E., Lambert, P. R., Goldstein, B. J., Forge, A. \& Corwin, J. T. Regenerative proliferation in inner ear sensory epithelia from adult guinea pigs and humans. Science 259, 1619-1622 (1993).

7. Forge, A., Li, L., Corwin, J. T. \& Nevill, G. Ultrastructural evidence for hair cell regeneration in the mammalian inner ear. Science 259, 1616-1619 (1993).

8. Balak, K. J., Corwin, J. T. \& Jones, J. E. Regenerated hair cells can originate from supporting cell progeny: evidence from phototoxicity and laser ablation experiments in the lateral line system. J. Neurosci. 10, 2502-2512 (1990).

9. White, P. M., Doetzlhofer, A., Lee, Y. S., Groves, A. K. \& Segil, N. Mammalian cochlear supporting cells can divide and trans-differentiate into hair cells. Nature 441, 984-987 (2006)

10. Monzack, E. L. \& Cunningham, L. L. Lead roles for supporting actors: critical functions of inner ear supporting cells. Hear Res 303, 20-29 (2013).

11. Sage, C. et al. Proliferation of functional hair cells in vivo in the absence of the retinoblastoma protein. Science 307, 1114-1118 (2005).

12. Chen, P. \& Segil, N. p27(Kip1) links cell proliferation to morphogenesis in the developing organ of Corti. Development 126, 1581-1590 (1999).

13. Chen, $P$. et al. Progressive hearing loss in mice lacking the cyclin-dependent kinase inhibitor Ink4d. Nat. Cell Biol. 5, 422-426 (2003).

14. Huang, M. et al. Overlapping and distinct $\mathrm{pRb}$ pathways in the mammalian auditory and vestibular organs. Cell Cycle 10, 337-351 (2011). 
15. Laine, H. et al. p19(Ink4d) and p21(Cip1) collaborate to maintain the postmitotic state of auditory hair cells, their codeletion leading to DNA damage and p53-mediated apoptosis. J. Neurosci. 27, 1434-1444 (2007).

16. Oesterle, E. C., Chien, W. M., Campbell, S., Nellimarla, P. \& Fero, M. L. p27 (Kip1) is required to maintain proliferative quiescence in the adult cochlea and pituitary. Cell Cycle 10, 1237-1248 (2011).

17. Zheng, J. L. \& Gao, W. Q. Overexpression of Math1 induces robust production of extra hair cells in postnatal rat inner ears. Nat. Neurosci. 3, 580-586 (2000).

18. Kawamoto, K., Ishimoto, S., Minoda, R., Brough, D. E. \& Raphael, Y. Math1 gene transfer generates new cochlear hair cells in mature guinea pigs in vivo. $J$. Neurosci. 23, 4395-4400 (2003).

19. Izumikawa, M. et al. Auditory hair cell replacement and hearing improvement by Atoh1 gene therapy in deaf mammals. Nat. Med 11, 271-276 (2005).

20. Kelly, M. C., Chang, Q., Pan, A., Lin, X. \& Chen, P. Atoh1 directs the formation of sensory mosaics and induces cell proliferation in the postnatal mammalian cochlea in vivo. J. Neurosci. 32, 6699-6710 (2012).

21. Liu, Z. et al. Age-dependent in vivo conversion of mouse cochlear pillar and Deiters' cells to immature hair cells by Atohl ectopic expression. J. Neurosci. 32, 6600-6610 (2012).

22. Yang, S. M. et al. Regeneration of stereocilia of hair cells by forced Atoh1 expression in the adult mammalian cochlea. PLoS ONE 7, e46355 (2012).

23. Lee, S. G. et al. Myc and Fgf Are Required for Zebrafish Neuromast Hair Cell Regeneration. PLoS ONE 11, e0157768 (2016).

24. Pan, W., Jin, Y., Stanger, B. \& Kiernan, A. E. Notch signaling is required for the generation of hair cells and supporting cells in the mammalian inner ear. Proc. Natl. Acad. Sci. USA 107, 15798-15803 (2010).

25. Hartman, B. H., Reh, T. A. \& Bermingham-McDonogh, O. Notch signaling specifies prosensory domains via lateral induction in the developing mammalian inner ear. Proc. Natl. Acad. Sci. USA 107, 15792-15797 (2010).

26. Ma, E. Y., Rubel, E. W. \& Raible, D. W. Notch signaling regulates the extent of hair cell regeneration in the zebrafish lateral line. J. Neurosci. 28, 2261-2273 (2008).

27. Stadtfeld, M., Nagaya, M., Utikal, J., Weir, G. \& Hochedlinger, K. Induced pluripotent stem cells generated without viral integration. Science 322, 945-949 (2008).

28. Shu, Y. et al. Identification of adeno-associated viral vectors that target neonatal and adult mammalian inner ear cell subtypes. Hum. Gene Ther. 27, 687-699 (2016).

29. Murtaugh, L. C., Stanger, B. Z., Kwan, K. M. \& Melton, D. A. Notch signaling controls multiple steps of pancreatic differentiation. Proc. Natl Acad. Sci. USA 100, 14920-14925 (2003).

30. Lin, C. Y. et al. Transcriptional amplification in tumor cells with elevated cMyc. Cell 151, 56-67 (2012).

31. Kolev, V. et al. The intracellular domain of Notch ligand Deltal induces cell growth arrest. FEBS Lett. 579, 5798-5802 (2005).

32. Arnold, K. et al. Sox $2(+)$ adult stem and progenitor cells are important for tissue regeneration and survival of mice. Cell Stem Cell 9, 317-329 (2011).

33. Mizutari, K. et al. Notch inhibition induces cochlear hair cell regeneration and recovery of hearing after acoustic trauma. Neuron 77, 58-69 (2013).

34. Ahmed, M. et al. Eyal-Sixl interaction is sufficient to induce hair cell fate in the cochlea by activating Atoh1 expression in cooperation with Sox2. Dev. Cell 22, 377-390 (2012).

35. Zheng, J. L., Shou, J., Guillemot, F., Kageyama, R. \& Gao, W. Q. Hes1 is a negative regulator of inner ear hair cell differentiation. Development 127, $4551-4560$ (2000).

36. Luo, X. J. et al. GATA3 controls the specification of prosensory domain and neuronal survival in the mouse cochlea. Hum. Mol. Genet 22, 3609-3623 (2013).

37. Zine, A. \& Romand, R. Development of the auditory receptors of the rat: a SEM study. Brain Res 721, 49-58 (1996).

38. Goodyear, R. J. et al. A receptor-like inositol lipid phosphatase is required for the maturation of developing cochlear hair bundles. J. Neurosci. 23, 9208-9219 (2003).

39. Meyers, J. R. et al. Lighting up the senses: FM1-43 loading of sensory cells through nonselective ion channels. J. Neurosci. 23, 4054-4065 (2003).

40. Chan, S. M., Weng, A. P., Tibshirani, R., Aster, J. C. \& Utz, P. J. Notch signals positively regulate activity of the mTOR pathway in T-cell acute lymphoblastic leukemia. Blood 110, 278-286 (2007).

41. Gera, J. F. et al. AKT activity determines sensitivity to mammalian target of rapamycin (mTOR) inhibitors by regulating cyclin D1 and c-myc expression. J. Biol. Chem. 279, 2737-2746 (2004).

42. Masui, K. et al. mTOR complex 2 controls glycolytic metabolism in glioblastoma through FoxO acetylation and upregulation of c-Myc. Cell Metab. 18, 726-739 (2013)

43. Lamming, D. W. Inhibition of the Mechanistic Target of Rapamycin (mTOR)-Rapamycin and Beyond. Cold Spring Harb Perspect Med 6, a025924 (2016).
44. Choi, Y. J. et al. Inhibitory effect of mTOR activator MHY1485 on autophagy: suppression of lysosomal fusion. PLoS ONE 7, e43418 (2012).

45. Morton, C. C. \& Nance, W. E. Newborn hearing screening-a silent revolution. N. Engl. J. Med 354, 2151-2164 (2006).

46. Li, H., Liu, H. \& Heller, S. Pluripotent stem cells from the adult mouse inner ear. Nat. Med 9, 1293-1299 (2003).

47. Walters, B. J. et al. In Vivo Interplay between p27(Kip1), GATA3, ATOH1, and POU4F3 Converts Non-sensory Cells to Hair Cells in Adult Mice. Cell Rep. 19, 307-320 (2017).

48. Dominguez-Frutos, E. et al. N-myc controls proliferation, morphogenesis, and patterning of the inner ear. J. Neurosci. 31, 7178-7189 (2011).

49. Burns, J. C., Yoo, J. J., Atala, A. \& Jackson, J. D. MYC gene delivery to adult mouse utricles stimulates proliferation of postmitotic supporting cells in vitro. PLoS ONE 7, e48704 (2012).

50. Belin, S. et al. Injury-induced decline of intrinsic regenerative ability revealed by quantitative proteomics. Neuron 86, 1000-1014 (2015)

51. Seal, R. P. et al. Sensorineural deafness and seizures in mice lacking vesicular glutamate transporter 3. Neuron 57, 263-275 (2008).

52. Li, W. et al. Notch inhibition induces mitotically generated hair cells in mammalian cochleae via activating the Wnt pathway. Proc. Natl Acad. Sci. USA 112, 166-171 (2015).

53. Su, X. et al. Abnormal expression of REST/NRSF and Myc in neural stem/ progenitor cells causes cerebellar tumors by blocking neuronal differentiation. Mol. Cell Biol. 26, 1666-1678 (2006).

54. Liu, Z., Fang, J., Dearman, J., Zhang, L. \& Zuo, J. In vivo generation of immature inner hair cells in neonatal mouse cochleae by ectopic Atoh1 expression. PLoS ONE 9, e89377 (2014).

55. Morita, M. et al. mTOR coordinates protein synthesis, mitochondrial activity and proliferation. Cell Cycle 14, 473-480 (2015).

56. Saxton, R. A. \& Sabatini, D. M. mTOR signaling in growth, metabolism, and disease. Cell 169, 361-371 (2017)

57. Gregorian, C. et al. Pten deletion in adult neural stem/progenitor cells enhances constitutive neurogenesis. J. Neurosci. 29, 1874-1886 (2009).

58. Park, K. K. et al. Promoting axon regeneration in the adult CNS by modulation of the PTEN/mTOR pathway. Science 322, 963-966 (2008)

59. Montcouquiol, M. \& Corwin, J. T. Intracellular signals that control cell proliferation in mammalian balance epithelia: key roles for phosphatidylinositol-3 kinase, mammalian target of rapamycin, and S6 kinases in preference to calcium, protein kinase $\mathrm{C}$, and mitogen-activated protein kinase. J. Neurosci. 21, 570-580 (2001).

60. Bhatt, K. A., Liberman, M. C. \& Nadol, J. B. Jr. Morphometric analysis of agerelated changes in the human basilar membrane. Ann. Otol. Rhinol. Laryngol. 110, 1147-1153 (2001).

61. Izumikawa, M., Batts, S. A., Miyazawa, T., Swiderski, D. L. \& Raphael, Y. Response of the flat cochlear epithelium to forced expression of Atoh1. Hear. Res. 240, 52-56 (2008).

62. Sergeyenko, Y., Lall, K., Liberman, M. C. \& Kujawa, S. G. Age-related cochlear synaptopathy: an early-onset contributor to auditory functional decline. J. Neurosci. 33, 13686-13694 (2013).

63. Okamoto, Y. et al. Permanent threshold shift caused by acute cochlear mitochondrial dysfunction is primarily mediated by degeneration of the lateral wall of the cochlea. Audio. Neurootol. 10, 220-233 (2005).

64. Doherty, J. K. \& Linthicum, F. H. Jr. Spiral ligament and stria vascularis changes in cochlear otosclerosis: effect on hearing level. Otol. Neurotol. 25, 457-464 (2004)

65. Zinin, N. et al. MYC proteins promote neuronal differentiation by controlling the mode of progenitor cell division. EMBO Rep. 15, 383-391 (2014).

66. Orian, A. et al. A Myc-Groucho complex integrates EGF and Notch signaling to regulate neural development. Proc. Natl. Acad. Sci. USA 104, 15771-15776 (2007).

67. Magnusson, J. P. et al. A latent neurogenic program in astrocytes regulated by Notch signaling in the mouse. Science 346, 237-241 (2014).

68. Allen, T. D., Rodriguez, E. M., Jones, K. D. \& Bishop, J. M. Activated Notch1 induces lung adenomas in mice and cooperates with Myc in the generation of lung adenocarcinoma. Cancer Res 71, 6010-6018 (2011).

69. Stanger, B. Z., Datar, R., Murtaugh, L. C. \& Melton, D. A. Direct regulation of intestinal fate by Notch. Proc. Natl Acad. Sci. USA 102, 12443-12448 (2005).

\section{Acknowledgements}

We are grateful to David Corey for critical reading of the manuscript. We would like to thank Shan Sun, Sofia Chen for help with the figures, Haobing Wang for help with the movies and Corena Loeb for editing the manuscript. The authors acknowledge funding from NIH R01DC006908, R56DC006908 (Z-Y.C.), DOD W81XWH1810331 (Z-Y.C.), Fredrick and Ines Yeatts hair cell regeneration fellowship (Z-Y.C.) and David-Shulsky Foundation (Z-Y.C.), the Major State Basic Research Development Program of China (2011CB504506)(Z.W.), (2011CB504500)(H.L.), Key Laboratory of Hearing Medicine, 
National Health and Family Planning Commission, Shanghai, China (Z.W.; H.L.), the National Natural Science Foundation of China (No. 81230019)(H.L.) (No. 81822011, 81771013) (Y.S.), Science and Technology Commission of Shanghai Municipality (17ZR1448600, 18410712400) (Y.S.), NIH R01DC005575, R01DC012115 (X.L.) and NIH R01DC017166 (A.A.I.).

\section{Author contributions}

Y.S, W.L, M.H., and Y.Z.Q. designed and performed experiments, analyzed data, and interpreted results. Y.T, D.S., C.T., and A.A.I. performed experiments and analyzed data. X.L, K.H., and W.Z analyzed data. H.L designed experiments and analyzed data. Z-Y.C. conceived the project, designed experiments, analyzed data, interpreted results and wrote the paper. All authors edited the paper.

\section{Competing interests}

Patent applications based on the work have been filed (Z-Y.C., Y.Q., and W.L.). The remaining authors declare no competing interests.

\section{Additional information}

Supplementary information is available for this paper at https://doi.org/10.1038/s41467019-13157-7.

Correspondence and requests for materials should be addressed to Z.-Y.C.
Peer review information Nature Communications thanks the anonymous reviewer(s) for their contribution to the peer review of this work. Peer reviewer reports are available.

Reprints and permission information is available at http://www.nature.com/reprints

Publisher's note Springer Nature remains neutral with regard to jurisdictional claims in published maps and institutional affiliations.

\section{cc) (i)}

Open Access This article is licensed under a Creative Commons Attribution 4.0 International License, which permits use, sharing, adaptation, distribution and reproduction in any medium or format, as long as you give appropriate credit to the original author(s) and the source, provide a link to the Creative Commons license, and indicate if changes were made. The images or other third party material in this article are included in the article's Creative Commons license, unless indicated otherwise in a credit line to the material. If material is not included in the article's Creative Commons license and your intended use is not permitted by statutory regulation or exceeds the permitted use, you will need to obtain permission directly from the copyright holder. To view a copy of this license, visit http://creativecommons.org/ licenses/by/4.0/.

(C) The Author(s) 2019 